\title{
Carbonylative Sonogashira Coupling of Terminal Alkynes with Aqueous Ammonia
}

\author{
Mohamed S. Mohamed Ahmed and Atsunori Mori* \\ Chemical Resources Laboratory, Tokyo Institute of Technology, 4259 Nagatsuta, \\ Yokohama 226-8503, Japan.
}

\section{Supporting Information}

\section{Experimental}

General poecedure for the carbonylative coupling of phenylethyne (1) with an aryl iodide (2) summarized in Table 1: To a $25 \mathrm{~mL}$ of Schlenk tube equipped with a magnetic stirring bar under argon were added $\mathrm{PdCl}_{2}\left(\mathrm{PPh}_{3}\right)_{2}(3.5 \mathrm{mg}, 0.005 \mathrm{mmol})$ and THF $(3 \mathrm{~mL})$. Phenylethyne $(66 \mu \mathrm{L}, 0.6 \mathrm{mmol})$ and $2(0.5 \mathrm{mmol})$ were added successively to the mixture to form a pale yellow solution. Then, aqueous ammonia ( 0.5 $\mathrm{M}, 2 \mathrm{~mL}, 1.0 \mathrm{mmol}$ ) was added dropwise via syringe. The atmosphere was replaced with carbon monoxide with a balloon and stirring was continued at room temperature. After the period shown in Table 1, the mixture was passed through a Celite pad and the filtrate was washed with brine. The aqueous layer was extracted with diethyl ether $(3 \times 15 \mathrm{~mL})$ and the combined organic layers were dried over anhydrous $\mathrm{MgSO}_{4}$, and concentrated in vacuo. The residue was purified by flash chromatography using hexanes-ethyl acetate to afford the corresponding $\alpha, \beta$-alkynyl ketones 3 .

1-(4-Methoxyphenyl)-3-phenyl-2-propynone (3a) ${ }^{1}$ : Reaction time: $41 \mathrm{~h}$. Purification by flash chromatography (30:1 hexanes:ethyl acetate) afforded the product in $72 \%$ yield ( 85 mg) as a colorless solid. Mp $98{ }^{\circ} \mathrm{C}$, Lit. mp $100{ }^{\circ} \mathrm{C}$. IR (KBr): $v=2201,1630 \mathrm{~cm}^{-1} .{ }^{1} \mathrm{H}$ NMR $\left(300 \mathrm{MHz}, \mathrm{CDCl}_{3}\right): \delta 3.90 \mathrm{ppm}(\mathrm{s}, 3 \mathrm{H}), 6.99(\mathrm{~d}, J=8.7 \mathrm{~Hz}, 2 \mathrm{H}), 7.38-7.50(\mathrm{~m}$, $3 \mathrm{H}), 7.67(\mathrm{dd}, J=8.1,1.5 \mathrm{~Hz}, 2 \mathrm{H}), 8.20(\mathrm{~d}, J=8.7 \mathrm{~Hz}, 2 \mathrm{H}) .{ }^{13} \mathrm{C}$ NMR $(300 \mathrm{MHz}$, $\left.\mathrm{CDCl}_{3}\right): \delta 55.54,86.85,92.27,113.83,120.26,128.60,130.22130 .55,131.93,132.90$, $164.43,176.63$.

1-(2-Methoxyphenyl)-3-phenyl-2-propynone (3b) ${ }^{2}$ : Reaction time: $24 \mathrm{~h}$. Purification by flash chromatography (50:1 hexanes:ethyl acetate) afforded the product in $76 \%$ yield (90 $\mathrm{mg}$ ) as a colorless oil. IR (neat): $v=2199,1641,1620,1597 \mathrm{~cm}^{-1} .{ }^{1} \mathrm{H}$ NMR $(300 \mathrm{MHz}$, $\left.\mathrm{CDCl}_{3}\right): \delta 3.97(\mathrm{~s}, 3 \mathrm{H}), 7.01-7.10(\mathrm{~m}, 2 \mathrm{H}), 7.36-7.76(\mathrm{~m}, 6 \mathrm{H}), 8.10(\mathrm{dd}, J=7.5,1.5 \mathrm{~Hz}$, $2 \mathrm{H}) .{ }^{13} \mathrm{C}$ NMR $\left(300 \mathrm{MHz}, \mathrm{CDCl}_{3}\right): 55.88,89.12,91.55,112.14,120.26,120.63,126.64$, $128.54,130.41,132.65,132.92,135.00,159.77,176.73$.

1-(3-Methoxyphenyl)-3-phenyl-2-propynone (3c): Reaction time: $51 \mathrm{~h}$. Purification by flash chromatography (50:1 hexanes:ethyl acetate) afforded the product in $81 \%$ yield (95 $\mathrm{mg}$ ) as a colorless oil. IR (neat): $v=2205,1642,1597,1582 \mathrm{~cm}^{-1} .{ }^{1} \mathrm{H}$ NMR $(300 \mathrm{MHz}$, $\mathrm{CDCl}_{3}$ ): $\delta 3.90$ (s, 3H), 7.18 (ddd, $\left.J=8.1,1.7,1.2 \mathrm{~Hz}, 1 \mathrm{H}\right), 7.40-7.53(\mathrm{~m}, 4 \mathrm{H}), 7.67-7.72$

\footnotetext{
${ }^{1}$ Johnston, K. M.; Shotter, R. G. J. Chem. Soc., C. 1967, 23, 2476.

${ }^{2}$ Larock, R. C.; Harrison, L. W. J. Am. Chem. Soc. 1984, 106, 4218.
} 
(m, 3H), 7.87 (ddd, $\left.J=7.8,1.5,1.2 \mathrm{~Hz}, 1 \mathrm{H}) .{ }^{13} \mathrm{C} \mathrm{NMR} \mathrm{(300} \mathrm{MHz,} \mathrm{CDCl}_{3}\right): 55.44,86.90$, 92.97, 112.72, 120.03, 120.94, 122.85, 128.65, 129.61, 130.79, 133.05, 138.17, 159.74., 177.75. HRMS (EI) $\mathrm{m} / \mathrm{z}$ calcd for $\mathrm{C}_{16} \mathrm{H}_{16} \mathrm{O}_{2}=236.0837$, found 236.0845 .

3-Phenyl-1-(4-methylphenyl)-2-propynone (3d) ${ }^{3}$ : Reaction time: $47 \mathrm{~h}$. Purification by flash chromatography (50:1 hexanes:ethyl acetate) afforded the product in $64 \%$ yield (70 $\mathrm{mg}$ ) as a colorless solid. $\mathrm{Mp} 70{ }^{\circ} \mathrm{C}$, Lit. $\mathrm{mp} 71{ }^{\circ} \mathrm{C}$. IR (KBr): $v=2203,1634,1608,1570$ $\mathrm{cm}^{-1} .{ }^{1} \mathrm{H}$ NMR $\left(300 \mathrm{MHz}, \mathrm{CDCl}_{3}\right): \delta 2.45 \mathrm{ppm}(\mathrm{s}, 3 \mathrm{H}), 7.30-7.45(\mathrm{~m}, 5 \mathrm{H}), 7.68(\mathrm{~d}, J=$ $, 8.7 \mathrm{~Hz}, 2 \mathrm{H}), 8.12(\mathrm{~d}, J=8.7 \mathrm{~Hz}, 2 \mathrm{H}) .{ }^{13} \mathrm{C}$ NMR $\left(300 \mathrm{MHz}, \mathrm{CDCl}_{3}\right): \delta 21.79,86.91$, $92.56,120.17,128.61,129.31,129.66,130.64,132.98,134.53,145.21,177.68$.

1,3-Diphenyl-2-propynone (3e) ${ }^{4}$ : Reaction time: $25 \mathrm{~h}$. Purification by flash chromatography (50:1 hexanes:ethyl acetate) afforded the product in $76 \%$ yield $(78 \mathrm{mg})$ as a colorless solid. Mp 46-47 ${ }^{\circ} \mathrm{C}$, Lit mp $46-48{ }^{\circ} \mathrm{C}$. IR (KBr): $v=2201,1651 \mathrm{~cm}^{-1} .{ }^{1} \mathrm{H}$ NMR (300 MHz, CDCl $)$ ): 7.40-7.56 (m, 5H), $7.60(\mathrm{~m}, 1 \mathrm{H}), 7.70$ (m, 2H), 8.23 (ddd, $J=$ $8.4,1.8,1.2 \mathrm{~Hz}, 2 \mathrm{H}) .{ }^{13} \mathrm{C}$ NMR $\left(300 \mathrm{MHz}, \mathrm{CDCl}_{3}\right): 86.85,93.11,120.09,128.61,128.67$, $129.57,130.80,133.06,134.12,136.84178 .03$.

1-(1-Nahpthyl)-3-phenyl-2-propynone (3f) $)^{5}$ : Reaction time: $34 \mathrm{~h}$. Purification by flash chromatography (50:1 hexanes:ethyl acetate) afforded the product in 50\% yield (64 $\mathrm{mg}$ ) as a colorless solid. Mp $95{ }^{\circ} \mathrm{C}$, Lit. $95{ }^{\circ} \mathrm{C}$. IR (KBr): $v=2193,1632,16.19 \mathrm{~cm}^{-1} .{ }^{1} \mathrm{H}$ NMR $\left(300 \mathrm{MHz}, \mathrm{CDCl}_{3}\right): \delta 7.43-7.71(\mathrm{~m}, 8 \mathrm{H}), 7.91(\mathrm{~d}, J=8.7 \mathrm{~Hz}, 1 \mathrm{H}), 8.11(\mathrm{~d}, J=8.4 \mathrm{~Hz}$, $1 \mathrm{H}), 8.65(\mathrm{~d}, J=6.9 \mathrm{~Hz}, 1 \mathrm{H}), 9.23(\mathrm{~d}, J=8.4 \mathrm{~Hz}, 1 \mathrm{H}) .{ }^{13} \mathrm{C} \mathrm{NMR}\left(300 \mathrm{MHz}, \mathrm{CDCl}_{3}\right)$ : $88.46,91.68,120.32,124.45,125.96,126.74,128.55,128.64,128.93,130.58,130.71$, $132.92,133.84,134.50,135.08,179.71$.

1-\{4-(1-Oxoethyl)phenyl\}-3-phenyl-2-propynone $\quad \mathbf{( 3 g ) :}$ Reaction time: $25 \mathrm{~h}$. Purification by flash chromatography (50:1 hexanes:ethyl acetate) afforded the product in $75 \%$ yield $(93 \mathrm{mg})$ as a colorless solid. Mp 103-104 ${ }^{\circ} \mathrm{C}$. IR $(\mathrm{KBr}): v=2199,1686,1632$ $\mathrm{cm}^{-1} .{ }^{1} \mathrm{H}$ NMR $\left(300 \mathrm{MHz}, \mathrm{CDCl}_{3}\right): \delta 2.67 \mathrm{ppm}(\mathrm{s}, 3 \mathrm{H}), 7.42-7.54(\mathrm{~m}, 3 \mathrm{H}), 7.69-7.73(\mathrm{~m}$, $2 \mathrm{H}), 8.09(\mathrm{~d}, J=8.7 \mathrm{~Hz}, 2 \mathrm{H}), 8.31(\mathrm{~d}, \mathrm{~J}=8.7 \mathrm{~Hz}, 2 \mathrm{H}) .{ }^{13} \mathrm{C} \mathrm{NMR}\left(300 \mathrm{MHz}, \mathrm{CDCl}_{3}\right): \delta$ $26.95,86.75,94.28,119.70,128.42,128.74,129.67,131.10,133.15,139.77,140.75$, 177.11, 197.42. HRMS (EI) $\mathrm{m} / \mathrm{z}$ calcd for $\mathrm{C}_{17} \mathrm{H}_{12} \mathrm{O}_{2}=248.0837$, found 248.0831 .

1-(4-Chlorophenyl)-3-phenyl-2-propynone (3i) ${ }^{6}$ : Reaction time: $12 \mathrm{~h}$. Purification by flash chromatography (50:1 hexanes:ethyl acetate) afforded the product in $67 \%$ yield ( 80 $\mathrm{mg}$ ) as a colorless solid. Mp 104-105 ${ }^{\circ} \mathrm{C}$, Lit. mp $105^{\circ} \mathrm{C}$. IR (KBr): $v=2201,1655 \mathrm{~cm}^{-1}$. ${ }^{1} \mathrm{H}$ NMR $\left(300 \mathrm{MHz}, \mathrm{CDCl}_{3}\right): \delta 7.43-7.51 \mathrm{ppm}(\mathrm{m}, 5 \mathrm{H}), 7.70(\mathrm{~d}, J=8.4 \mathrm{~Hz}, 2 \mathrm{H}), 8.16(\mathrm{~d}$, $\mathrm{J}=8.4 \mathrm{~Hz}, 2 \mathrm{H}) .{ }^{13} \mathrm{C} \mathrm{NMR}\left(300 \mathrm{MHz}, \mathrm{CDCl}_{3}\right): 86.52,93.59,119.80,128.68,128.94$, $130.81,130.93,133.05,135.21,140.65,176.59$.

\footnotetext{
${ }^{3}$ Baddar, F. G.; Al-Hajjar, F. H.; El-Rayyes, N. R. J. Heterocyclic Chem., 1976, 13, 195.

${ }^{4}$ Nahm, S.; Weinreb, S. M. Tetrahydron Lett. 1981, 22, 3815.

${ }^{5}$ Arcadi, A.; Cacchi, S.; Marinelli, F.; Pace, P.; Sanzi, G. Synlett, 1995, 823.

${ }^{6}$ Delaude, L.; Masdue, A. M.; Alper, H. Synthesis, 1994, 1149.
} 
General procedure for the carbonylative coupling of alkylalkynes 5 with aryl halides 2 summarized in Table 2: To a solution of an alkylalkyne $(5,0.6 \mathrm{mmol}), 2(0.5 \mathrm{mmol})$, $\mathrm{PdCl}_{2}\left(\mathrm{PPh}_{3}\right)_{2}(17.5 \mathrm{mg}, 0.025 \mathrm{mmol})$ and $\mathrm{CuI}(1.9 \mathrm{mg}, 0.01 \mathrm{mmol})$ in THF (3 mL) was added aqueous ammonia $(0.5 \mathrm{M}, 2 \mathrm{~mL}, 1.0 \mathrm{mmol})$. The atmosphere was replaced with carbon monoxide with a balloon and stirring was continued at room temperature for the period shown in Table 2. The resulting mixture was passed through a Celite pad and the filtrate was washed with brine. The aqueous layer was extracted with diethyl ether $(3 \times$ $15 \mathrm{~mL}$ ) and the combined organic layers were dried over $\mathrm{MgSO}_{4}$, and concentrated in vacuo. The residue was purified by flash chromatography on silica gel.

1-(4-Methoxyphenyl)-2-nonyn-1-one (6aa): Reaction time: $24 \mathrm{~h}$. Purification by flash chromatography (30:1 hexanes:ethyl acetate) afforded the product in 74\% yield $(90 \mathrm{mg})$ as a colorless oil. IR (neat): $v=2932,2858,2199,1638,1597 \mathrm{~cm}^{-1} .{ }^{1} \mathrm{H}$ NMR $(300 \mathrm{MHz}$, $\left.\mathrm{CDCl}_{3}\right): \delta 0.93 \mathrm{ppm}(\mathrm{t}, J=6.6 \mathrm{~Hz}, 3 \mathrm{H}), 1.31-1.68(\mathrm{~m}, 8 \mathrm{H}), 2.48(\mathrm{t}, J=6.9 \mathrm{~Hz}, 2 \mathrm{H}), 3.88$ $(\mathrm{s}, 3 \mathrm{H}), 6.94(\mathrm{~d}, J=8.4 \mathrm{~Hz}, 2 \mathrm{H}), 8.11(\mathrm{~d}, J=6.6 \mathrm{~Hz}, 2 \mathrm{H}) .{ }^{13} \mathrm{C} \mathrm{NMR}\left(300 \mathrm{MHz}, \mathrm{CDCl}_{3}\right)$ : $13.98,19.15,22.45,27.78,28.60,31.19,55.51,79.59$, 95.97, 113.65, 130.29, 131.86, 164.21, 176.95. HRMS (EI) m/z calcd for $\mathrm{C}_{16} \mathrm{H}_{20} \mathrm{O}_{2}=244.1463$, found 244.1461.

1-(4-Methylphenyl)-2-nonyn-1-one (6ad): Reaction time: $24 \mathrm{~h}$. Purification by flash chromatography (30:1 hexanes:ethyl acetate) afforded the product in 78\% yield $(89 \mathrm{mg})$ as a colorless oil. IR (neat): $v=2955,2936,2859,2199,1646,1605 \mathrm{~cm}^{-1} .{ }^{1} \mathrm{H}$ NMR (300 $\left.\mathrm{MHz} \mathrm{CDCl}_{3}\right): \delta 0.91 \mathrm{ppm}(\mathrm{t}, J=7.2 \mathrm{~Hz}, 3 \mathrm{H}), 1.20-1.75(\mathrm{~m}, 8 \mathrm{H}), 2.49(\mathrm{t}, J=7.2 \mathrm{~Hz}$, $2 \mathrm{H}), 7.27(\mathrm{~d}, J=8.7 \mathrm{~Hz}, 2 \mathrm{H}), 8.03(\mathrm{~d}, J=7.2 \mathrm{~Hz}, 2 \mathrm{H}) .{ }^{13} \mathrm{C} \mathrm{NMR}\left(300 \mathrm{MHz}, \mathrm{CDCl}_{3}\right): \delta$ 13.94, 19.13, 21.69, 22.42, 27.73, 28.56, 31.16, 79.67, 96.27, 129.12, 129.60, 134.60, 144.81, 177.90. HRMS (EI) m/z calcd for $\mathrm{C}_{16} \mathrm{H}_{20} \mathrm{O}=228.1514$, found 228.1511 .

1-(1-Naphthyl)-2-nonyn-1-one (6af): Reaction time: $25 \mathrm{~h}$. Purification by flash chromatography (30:1 hexanes:ethyl acetate) afforded the product in 60\% yield (79 $\mathrm{mg})$ as a colorless oil. IR (neat): $v=3050,2955,2936,2859,2209,1638 \mathrm{~cm}^{-1} .{ }^{1} \mathrm{H}$ NMR (300 $\left.\mathrm{MHz} \mathrm{CDCl}_{3}\right): \delta 0.91 \mathrm{ppm}(\mathrm{t}, J=6.9 \mathrm{~Hz}, 3 \mathrm{H}), 1.32-1.69(\mathrm{~m}, 8 \mathrm{H}), 2.51(\mathrm{t}, J=7.2 \mathrm{~Hz}$, 2H), 7.54-7.66 (m, 3H), 7.89 (d, $J=7.2 \mathrm{~Hz}, 1 \mathrm{H}), 8.06$ (d, $J=8.1 \mathrm{~Hz}, 1 \mathrm{H}), 8.54$ (dd, $J=$ 7.5, $1.5 \mathrm{~Hz}, 1 \mathrm{H}), 9.18(\mathrm{~d}, J=8.7 \mathrm{~Hz}, 1 \mathrm{H}) .{ }^{13} \mathrm{C}$ NMR $\left(300 \mathrm{MHz}, \mathrm{CDCl}_{3}\right): \delta 13.99,19.18$, $22.46,27.74,28.63,31.19,81.25,95.37,124.34,125.95,126.58,128.44,128.73,130.63$, 132.94, 133.75, 134.41, 134.76, 180.00. HRMS (EI) $\mathrm{m} / \mathrm{z}$ calcd for $\mathrm{C}_{19} \mathrm{H}_{20} \mathrm{O}=264.1514$, found 264.1518 .

1-\{4-(1-Oxoethyl)phenyl\}-2-nonyn-1-one (6ag): Reaction time: $30 \mathrm{~h}$. Purification by flash chromatography (30:1 hexanes:ethyl acetate) afforded the product in 47\% yield (60 $\mathrm{mg}$ ) as a colorless oil. IR (neat): $v=2967,2930,2859,2238,2203,1691,1649 \mathrm{~cm}^{-1} .{ }^{1} \mathrm{H}$ NMR $\left(300 \mathrm{MHz}, \mathrm{CDCl}_{3}\right): \delta 0.91 \mathrm{ppm}(\mathrm{t}, J=6.9 \mathrm{~Hz}, 3 \mathrm{H}), 1.20-1.75(\mathrm{~m}, 8 \mathrm{H}), 2.53(\mathrm{t}, J=$ $7.8 \mathrm{~Hz}, 2 \mathrm{H}), 2.66(\mathrm{~s}, 3 \mathrm{H}), 8.04(\mathrm{~d}, J=6.6 \mathrm{~Hz}, 2 \mathrm{H}), 8.22(\mathrm{~d}, J=6.6 \mathrm{~Hz}, 2 \mathrm{H}) .{ }^{13} \mathrm{C} \mathrm{NMR}$ $\left(300 \mathrm{MHz}, \mathrm{CDCl}_{3}\right): \delta 13.99,19.23,22.44,26.95,27.65,28.60,31.16,79.57,98.27$, 128.29, 129.64, 139.82, 140.56, 177.33, 197.51. HRMS (EI) $\mathrm{m} / \mathrm{z}$ calcd for $\mathrm{C}_{17} \mathrm{H}_{20} \mathrm{O}_{2}=$ 256.1463 , found 256.1474 . 
1-(2-Aminophenyl)-2-nonyn-1-one (6ah): Reaction time: $40 \mathrm{~h}$. Purification by flash chromatography (30:1 hexanes:ethyl acetate) afforded the product in $71 \%$ yield $(81 \mathrm{mg})$ as a pale yellow oil. IR (neat): $v=3449,3341,2955,2930,2859,2226,1622,1586 \mathrm{~cm}^{-1}$. ${ }^{1} \mathrm{H}$ NMR $\left(300 \mathrm{MHz}, \mathrm{CDCl}_{3}\right): \delta 0.90 \mathrm{ppm}(\mathrm{t}, J=6.9 \mathrm{~Hz}, 3 \mathrm{H}), 1.25-1.72(\mathrm{~m}, 8 \mathrm{H}), 2.48(\mathrm{t}, J$ $=7.8 \mathrm{~Hz}, 2 \mathrm{H}), 6.29$ (brs, 2H), 6.61-6.71 (m, 2H), 7.29-7.31 (m, 1H), 8.06-8.09 (m, 1H). ${ }^{13} \mathrm{C}$ NMR $\left(300 \mathrm{MHz}, \mathrm{CDCl}_{3}\right): \delta 13.93,19.08,22.40,27.73,28.53,31.13,79.75,95.77$, $115.80,116.58,118.68,134.53,134.92,150.89,179.83$. HRMS (EI) $\mathrm{m} / \mathrm{z}$ calcd for $\mathrm{C}_{15} \mathrm{H}_{19} \mathrm{ON}=229.1467$, found 229.1474. Anal. Calcd for $\mathrm{C}_{15} \mathrm{H}_{19} \mathrm{ON}$ : C, 78.56; H, 8.35; N, 6.11. Found: C, 78.76; H, 8.28; N, 5.99.

1-(4-Chlorophenyl)-2-nonyn-1-one (6ai): Reaction time: $26 \mathrm{~h}$. Purification by flash chromatography (30:1 hexanes:ethyl acetate) afforded the product in 70\% yield $(87 \mathrm{mg})$ as a colorless oil. IR (neat): $v=2930,2859,2238,2201,1637,1597,1576 \mathrm{~cm}^{-1} .{ }^{1} \mathrm{H}$ NMR $\left(300 \mathrm{MHz}, \mathrm{CDCl}_{3}\right): \delta 0.91(\mathrm{~s}, 3 \mathrm{H}), 1.20-1.75(\mathrm{~m}, 8 \mathrm{H}), 2.50(\mathrm{t}, J=7.2 \mathrm{~Hz}, 2 \mathrm{H}), 7.44(\mathrm{~d}, J$ $=8.1 \mathrm{~Hz}, 2 \mathrm{H}), 8.05(\mathrm{~d}, J=8.1 \mathrm{~Hz}, 2 \mathrm{H}) .{ }^{13} \mathrm{C} \mathrm{NMR}\left(300 \mathrm{MHz}, \mathrm{CDCl}_{3}\right): 13.95,19.16$, 22.42, 27.66, 28.58, 31.14, 79.33, 97.45, 128.77, 130.78, 135.27, 140.37, 176.81 HRMS (EI) $\mathrm{m} / \mathrm{z}$ calcd for $\mathrm{C}_{15} \mathrm{H}_{17} \mathrm{ClO}=248.0968$, found 248.0967 .

1,4-Di(1-oxo-2-nonyn-1-yl)benzene (8): Reaction time: $27 \mathrm{~h}$. Purification by flash chromatography (30:1 hexanes:ethyl acetate) afforded the product in 53\% yield $(93 \mathrm{mg})$ as a colorless oil. IR (neat): $v=2957,2930,2859,2236,2199,1657 \mathrm{~cm}^{-1} .{ }^{1} \mathrm{H}$ NMR (300 $\left.\mathrm{MHz} \mathrm{CDCl}_{3}\right): \delta 0.91 \mathrm{ppm}(\mathrm{t}, J=7.2 \mathrm{~Hz}, 6 \mathrm{H}), 1.30-1.76(\mathrm{~m}, 16 \mathrm{H}), 2.53(\mathrm{t}, J=6.9 \mathrm{~Hz}$, $4 \mathrm{H}), 8.22(\mathrm{~s}, 4 \mathrm{H}) .{ }^{13} \mathrm{C} \mathrm{NMR}\left(300 \mathrm{MHz}, \mathrm{CDCl}_{3}\right): \delta 13.99,19.25,22.45,27.66,28.62$, $31.17,79.63$, 98.40, 129.47, 140.33, 177.33. HRMS (EI) $\mathrm{m} / \mathrm{z}$ calcd for $\mathrm{C}_{24} \mathrm{H}_{30} \mathrm{O}_{2}=$ 350.2246 , found 350.2237 .

1-(1-Octyn-1-yl)-4-(1-oxo-2-nonyn-1-yl)benzene (9): Reaction time: 27 h. Purification by flash chromatography (30:1 hexanes:ethyl acetate) afforded the product in 37\% yield (60 mg) as a colorless oil. IR (neat): $v=2957,2932,2859,2234,2201,1647,1601 \mathrm{~cm}^{-1}$. ${ }^{1} \mathrm{H}$ NMR $\left(300 \mathrm{MHz}, \mathrm{CDCl}_{3}\right): \delta 0.90 \mathrm{ppm}(\mathrm{t}, J=6.9 \mathrm{~Hz}, 6 \mathrm{H}), 1.25-1.70(\mathrm{~m}, 16 \mathrm{H}), 2.40-$ $2.55(\mathrm{~m}, 4 \mathrm{H}), 7.46(\mathrm{~d}, J=8.7 \mathrm{~Hz}, 2 \mathrm{H}), 8.04(\mathrm{~d}, J=8.7 \mathrm{~Hz}, 2 \mathrm{H}) .{ }^{13} \mathrm{C}$ NMR $(300 \mathrm{MHz}$, $\left.\mathrm{CDCl}_{3}\right): \delta 14.00,14.03,19.21,19.55,22.47,22.52$. 27.72, 28.46, 28.59, 28.62, 31.19, $31.30,79.59,80.18,95.15,97.13,129.34,129.96,131.52,135.51,177.43$. HRMS (EI) $\mathrm{m} / \mathrm{z}$ calcd for $\mathrm{C}_{23} \mathrm{H}_{30} \mathrm{O}=322.2297$, found 322.2288 .

4,4-Dimethyl-1-(4-methoxyphenyl)-2-pentyn-1-one (6ba): Reaction time: $20 \mathrm{~h}$. Purification by flash chromatography (30:1 hexanes:ethyl acetate) afforded the product in $87 \%$ yield (94 mg) as a colorless oil. IR (neat): $v=2993,2932,2903,2842,2211,1647$, $1597,1576 \mathrm{~cm}^{-1} .{ }^{1} \mathrm{H}$ NMR $\left(300 \mathrm{MHz}, \mathrm{CDCl}_{3}\right): \delta 1.37(\mathrm{~s}, 9 \mathrm{H}), 3.88(\mathrm{~s}, 3 \mathrm{H}), 6.95(\mathrm{~d}, J=$ $8.4 \mathrm{~Hz}, 2 \mathrm{H}), 8.10(\mathrm{~d}, J=8.4 \mathrm{~Hz}, 2 \mathrm{H}) .{ }^{13} \mathrm{C} \mathrm{NMR}\left(300 \mathrm{MHz}, \mathrm{CDCl}_{3}\right): 27.95,30.18,55.50$, 78.02, 103.05, 113.66, 130.41, 131.83, 164.17, 177.06. HRMS (EI) $\mathrm{m} / \mathrm{z}$ calcd for $\mathrm{C}_{14} \mathrm{H}_{16} \mathrm{O}_{2}=216.1150$, found 216.1158 .

4,4-Dimethyl-1-phenyl-2-pentyn-1-one (6be) ${ }^{7}$ : Reaction time: 24 h. Purification by flash chromatography (30:1 hexanes:ethyl acetate) afforded the product in $77 \%$ yield (71

\footnotetext{
${ }^{7}$ Smith III, A. B.; Levenberg, P. A.; Suits, J. Z. Synthesis, 1986, 184.
} 
$\mathrm{mg}$ ) as a colorless oil. IR (neat): $v=3065,2973,2932,2870,2212,1647,1619,1599$, $1582 \mathrm{~cm}^{-1} .{ }^{1} \mathrm{H}$ NMR $\left(300 \mathrm{MHz}, \mathrm{CDCl}_{3}\right): \delta 1.39(\mathrm{~s}, 9 \mathrm{H}), 7.48-7.623(\mathrm{~m}, 3 \mathrm{H}), 8.12(\mathrm{dd}, J=$ 8.1, $1.2 \mathrm{~Hz}, 2 \mathrm{H}) .{ }^{13} \mathrm{C} \mathrm{NMR}\left(300 \mathrm{MHz}, \mathrm{CDCl}_{3}\right): 28.01,30.14,78.06,103.96,128.44$, $129.47,133.79,136.97,178.36$.

4,4-Dimethyl-1-(1-naphthyl)-2-pentyn-1-one (6bf): Reaction time: 19 h. Purification by flash chromatography (30:1 hexanes: ethyl acetate) afforded the product in $69 \%$ yield (82 $\mathrm{mg}$ ) as a colorless oil. IR (neat): $v=2973,2930,2869,2207,1637,1619,1591,1574 \mathrm{~cm}^{-}$ $1 .{ }^{1} \mathrm{H}$ NMR $\left(300 \mathrm{MHz}, \mathrm{CDCl}_{3}\right): \delta 1.40(\mathrm{~s}, 9 \mathrm{H}), 7.53-7.65(\mathrm{~m}, 3 \mathrm{H}), 7.88(\mathrm{~d}, J=7.2 \mathrm{~Hz}$, $1 \mathrm{H}), 8.06(\mathrm{~d}, J=8.1 \mathrm{~Hz}, 1 \mathrm{H}), 8.50(\mathrm{~d}, J=7.2 \mathrm{~Hz}, 1 \mathrm{H}), 9.15(\mathrm{~d}, J=8.7 \mathrm{~Hz}, 1 \mathrm{H}) .{ }^{13} \mathrm{C}$ NMR $\left(300 \mathrm{MHz}, \mathrm{CDCl}_{3}\right.$ ): 27.96, 30.14, 79.79, 102.31, 124.35, 125.96, 126.57, 128.43, 128.67, 130.64, 133.19, 133.76, 134.13, 134.66, 180.13. HRMS (EI) $\mathrm{m} / \mathrm{z}$ calcd for $\mathrm{C}_{17} \mathrm{H}_{16} \mathrm{O}=236.1201$, found 236.1208 .

4-Hydroxy-1-(4-methoxyphenyl)-4-methyl-2-pentyn-1-one (6ca): Reaction time: $46 \mathrm{~h}$. Purification by flash chromatography (30:1 hexanes:ethyl acetate) afforded the product in $55 \%$ yield based on ${ }^{1} \mathrm{H}$ NMR. Calculation was based on signals of methoxy groups at $3.89(\mathrm{~s}, 3 \mathrm{H}$ for $\mathbf{6 c a})$ and $3.78(\mathrm{~s}, 3 \mathrm{H}$ for $\mathbf{2 a})$.

5-Hydroxy-1-(4-methoxyphenyl)-2-pentyn-1-one (6da): Reaction time: $48 \mathrm{~h}$. Purification by flash chromatography (3:1 hexanes:ethyl acetate) afforded the product in $56 \%$ yield $(57 \mathrm{mg})$ as a colorless solid. Mp $104{ }^{\circ} \mathrm{C}$. IR (KBr): $v=3420,2204,1635 \mathrm{~cm}^{-1}$. ${ }^{1} \mathrm{H}$ NMR $\left(300 \mathrm{MHz}, \mathrm{CDCl}_{3}\right): \delta 2.44$ (brs, $\left.1 \mathrm{H}\right), 2.76(\mathrm{t}, J=6.3 \mathrm{~Hz}, 2 \mathrm{H}), 3.88(\mathrm{~s}, 3 \mathrm{H}), 3.89$ $(\mathrm{t}, J=6.3 \mathrm{~Hz}, 2 \mathrm{H}), 6.95(\mathrm{~d}, J=90 \mathrm{~Hz}, 2 \mathrm{H}), 8.11(\mathrm{~d}, J=9.0 \mathrm{~Hz}, 2 \mathrm{H}) .{ }^{13} \mathrm{C}$ NMR $(300$ $\left.\mathrm{MHz}, \mathrm{CDCl}_{3}\right): 23.52,55.54,60.27,80.65,92.62,113.78,129.93,132.03,164.48,176.90$. HRMS (EI) $\mathrm{m} / \mathrm{z}$ calcd for $\mathrm{C}_{12} \mathrm{H}_{12} \mathrm{O}_{3}=204.0786$, found 204.0794 . 


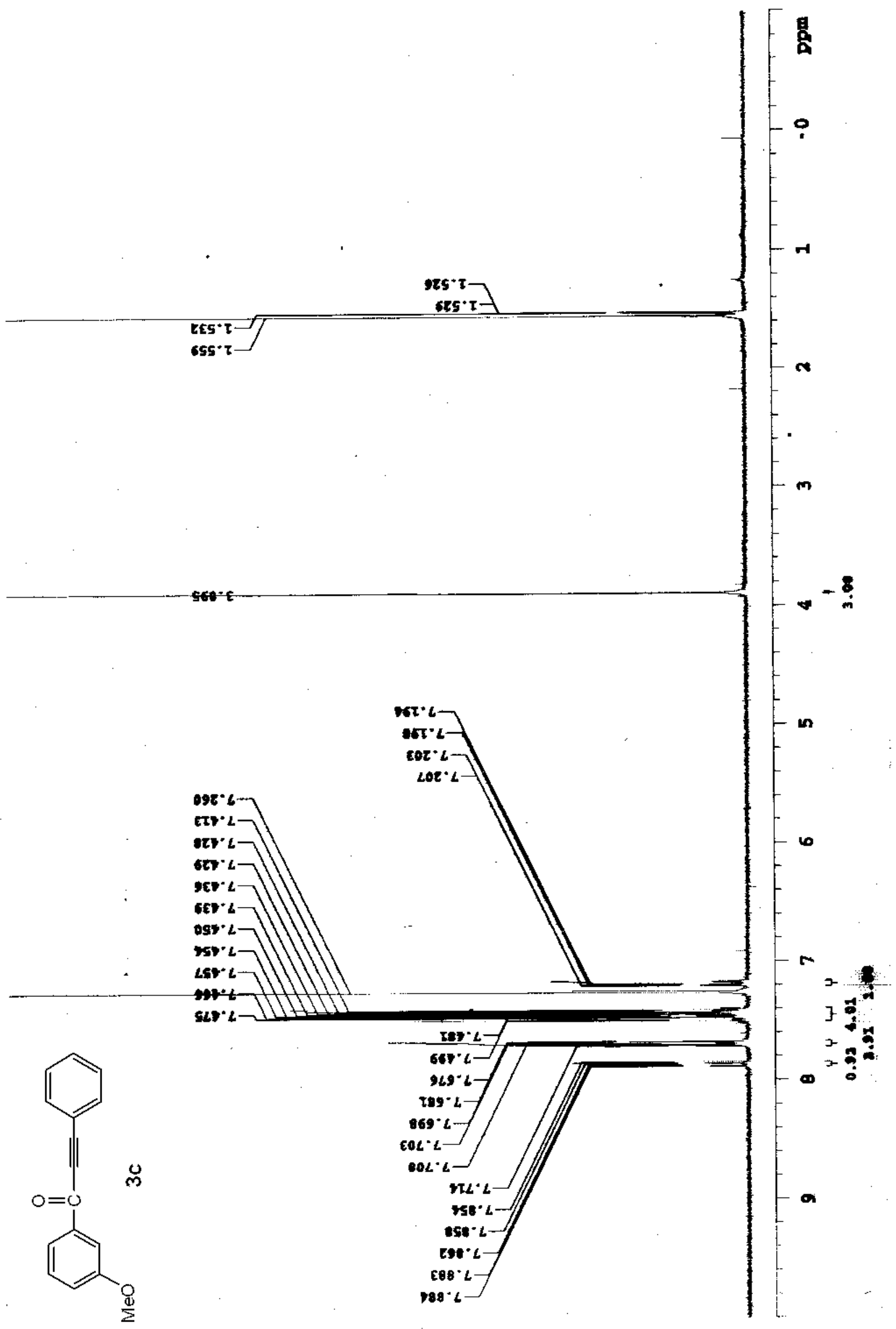




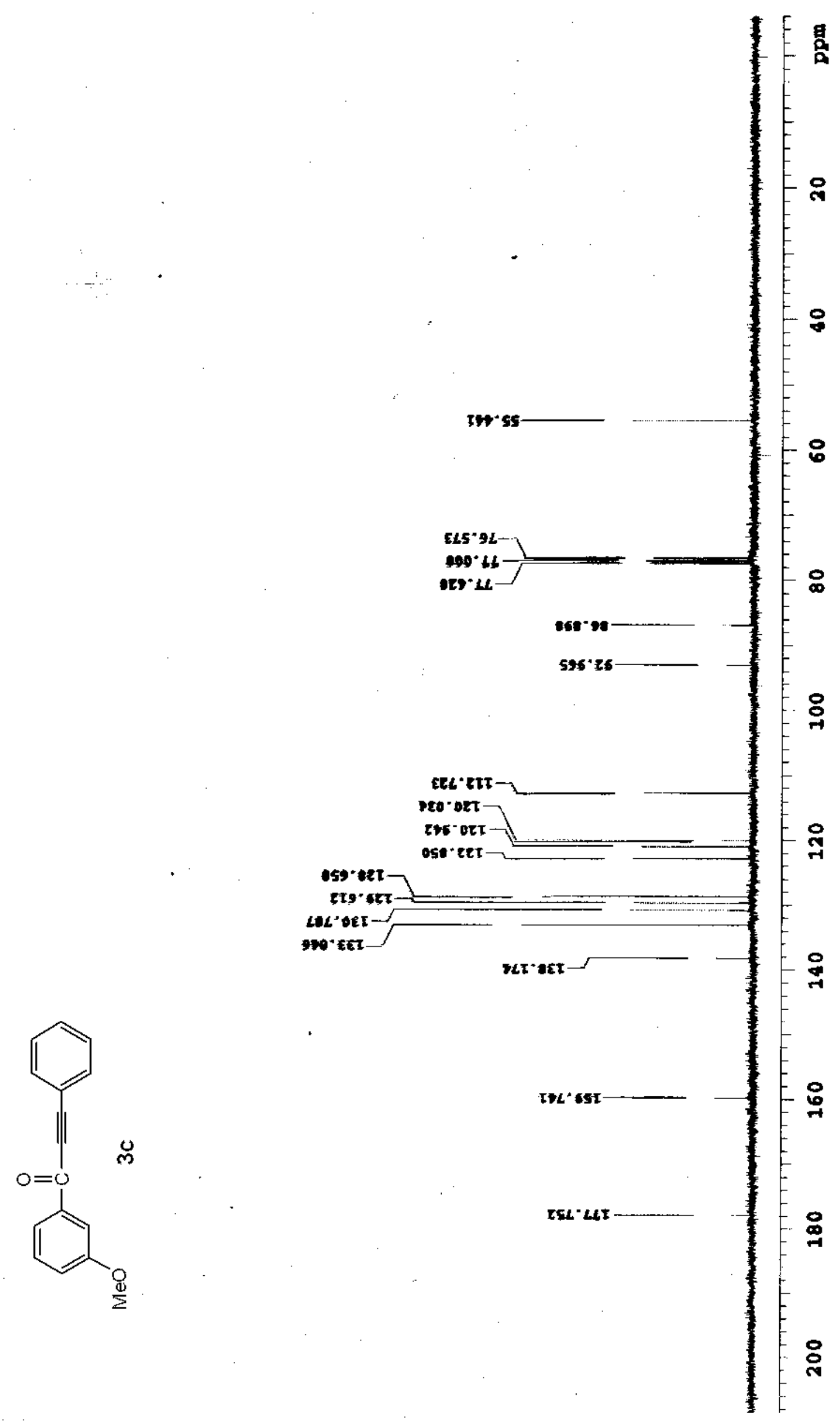




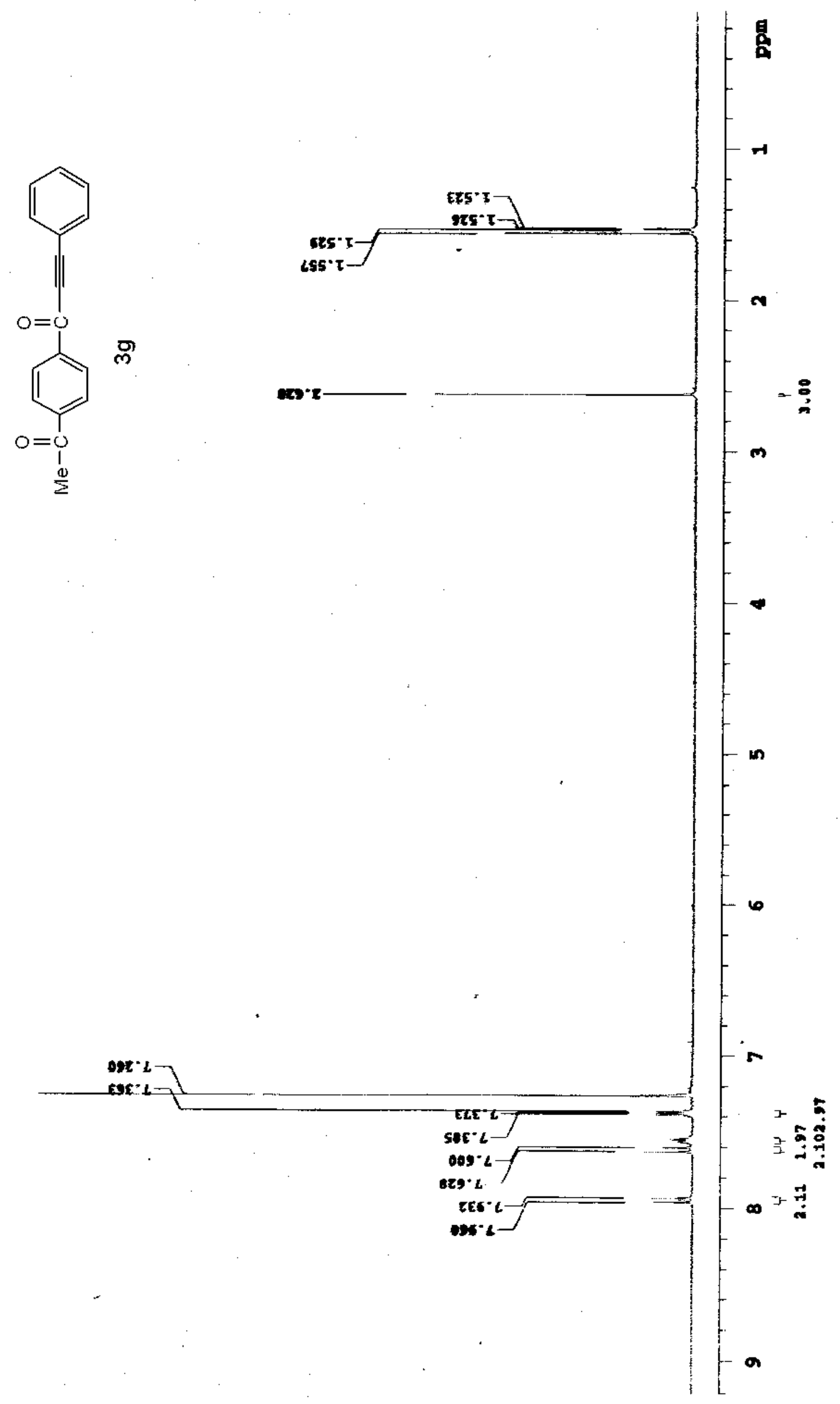




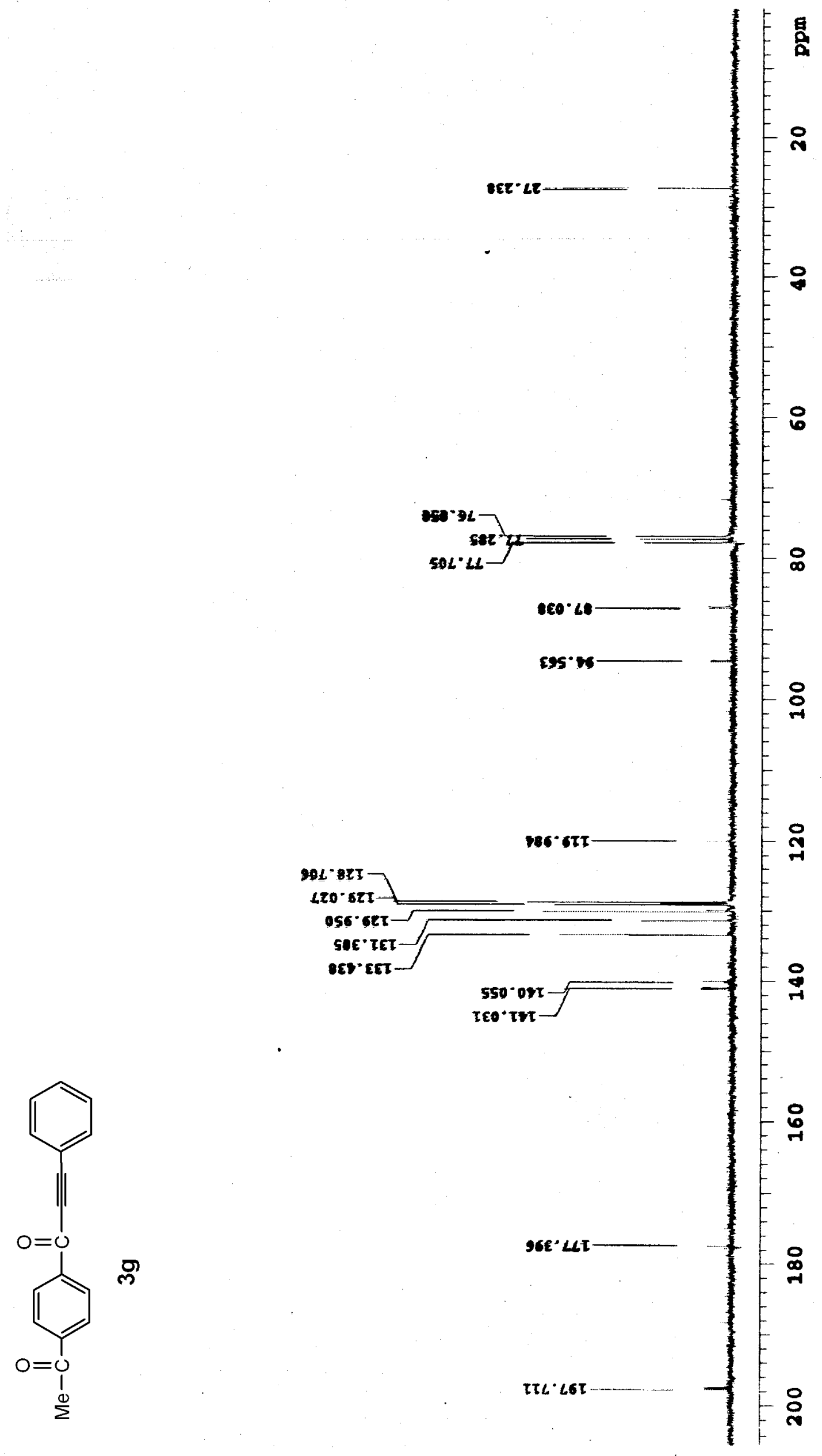




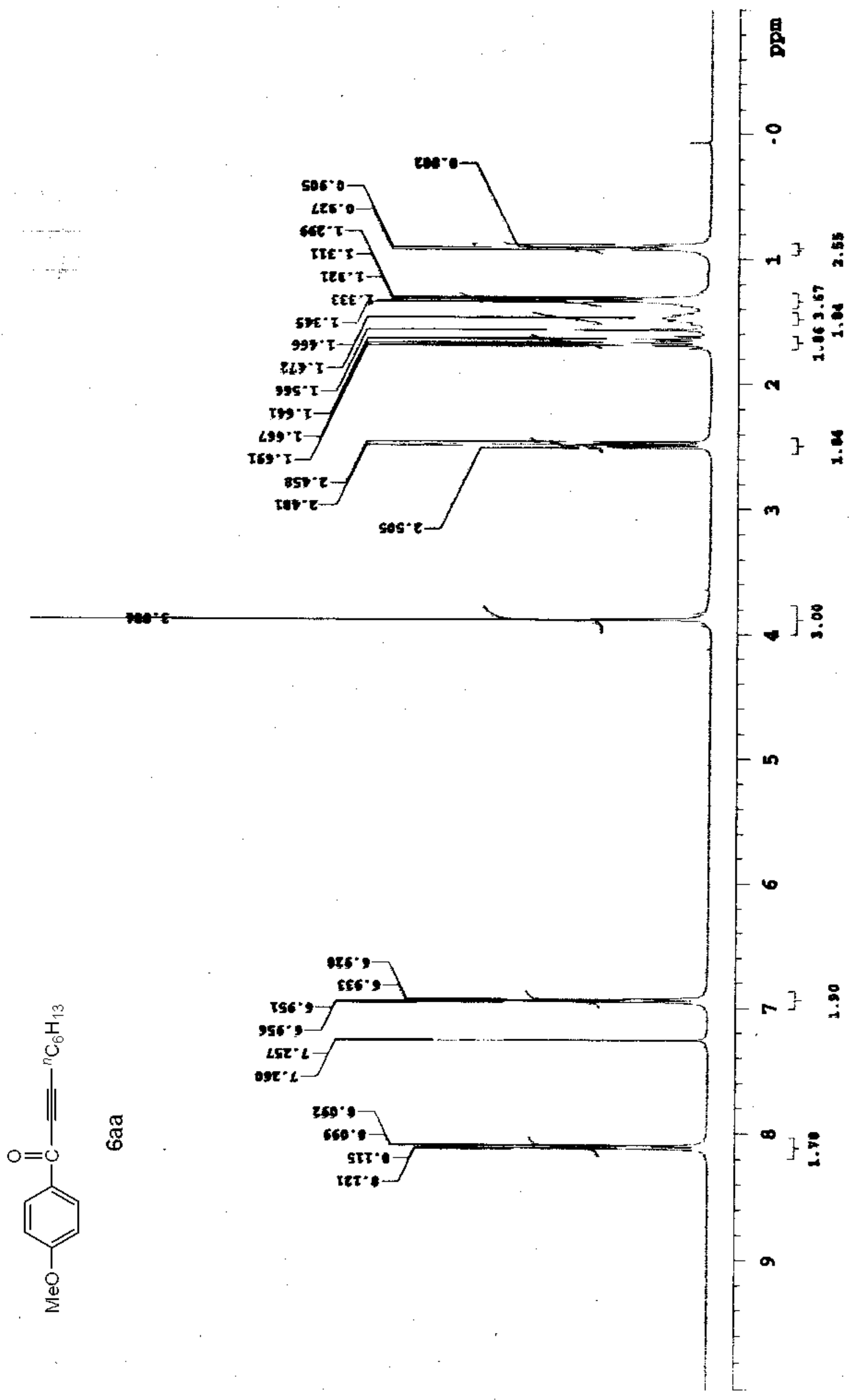




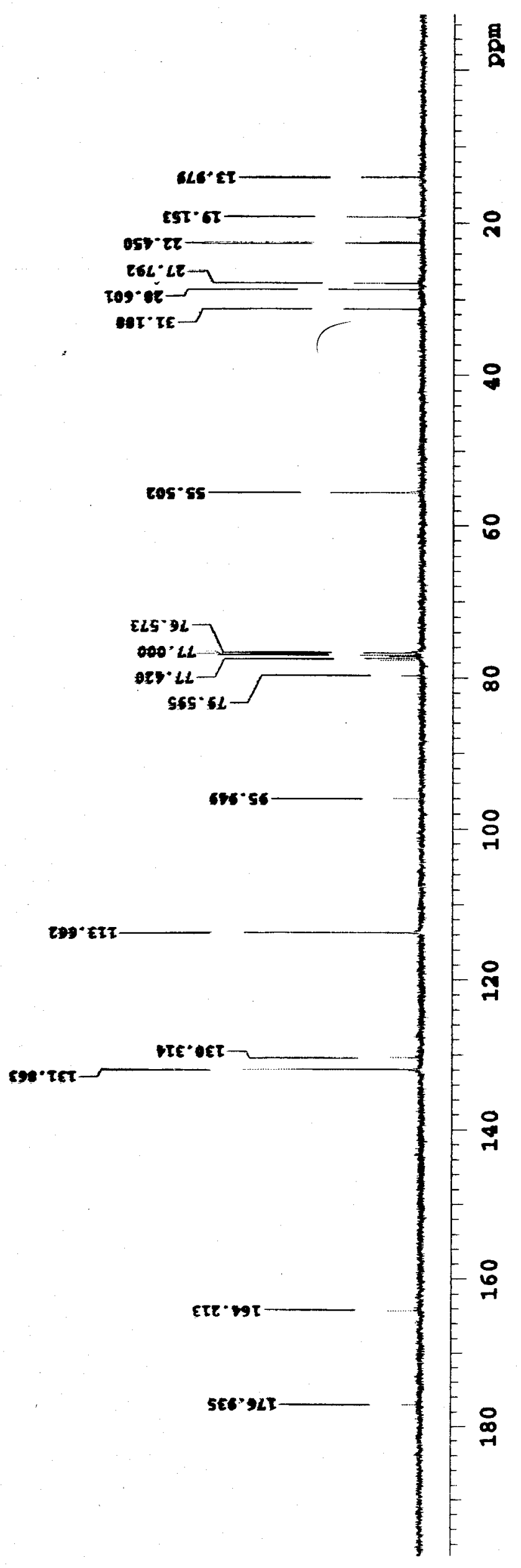




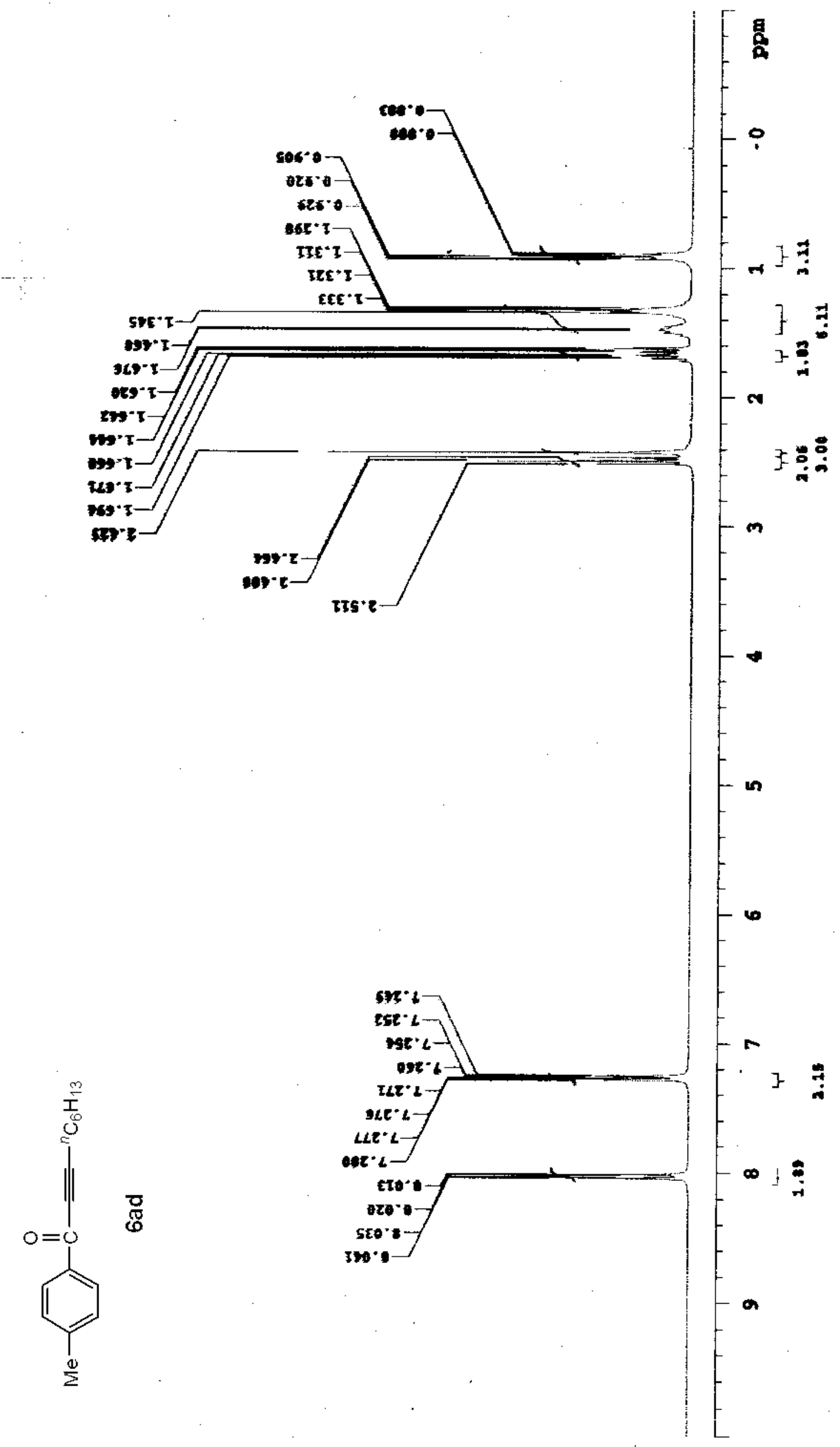




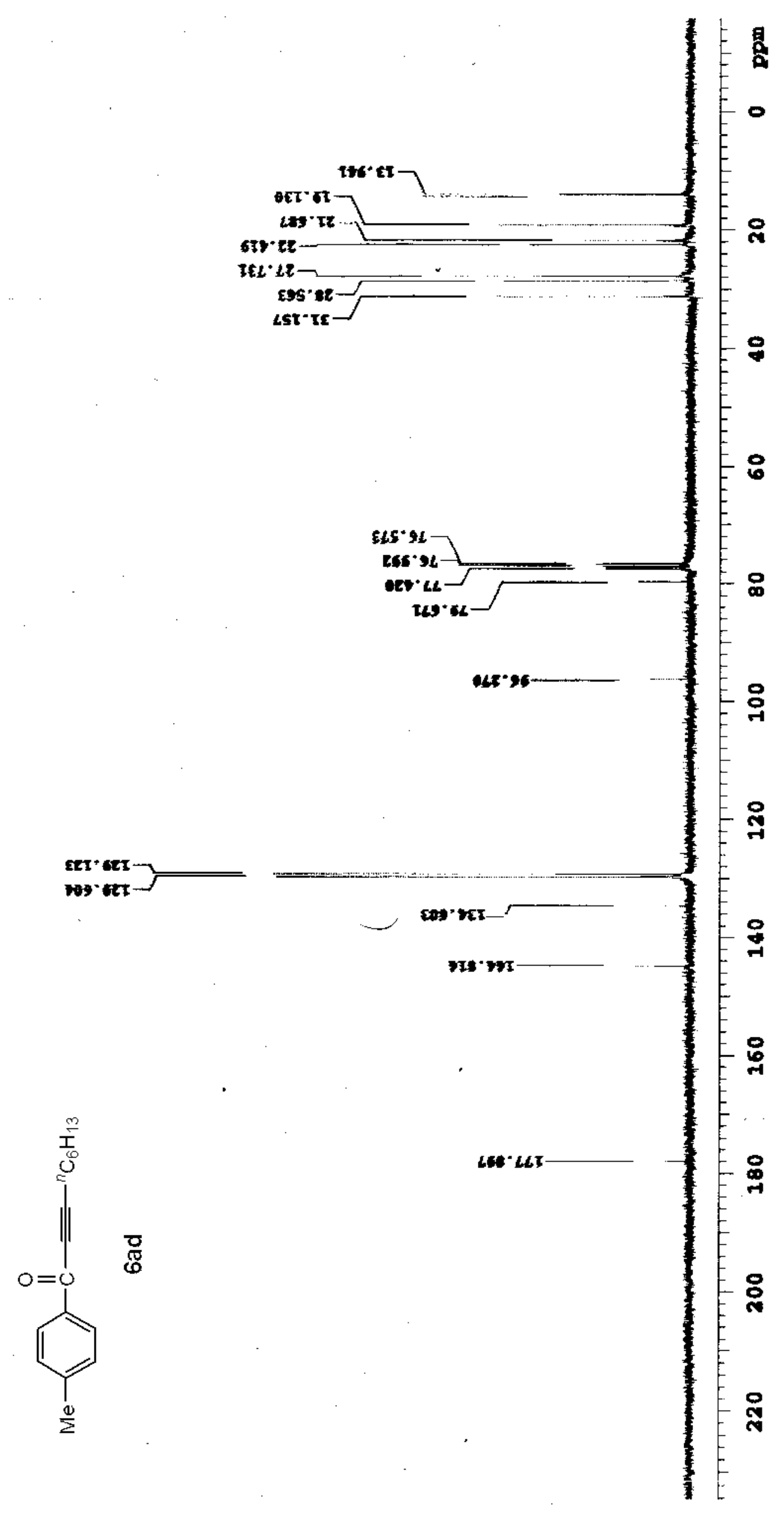




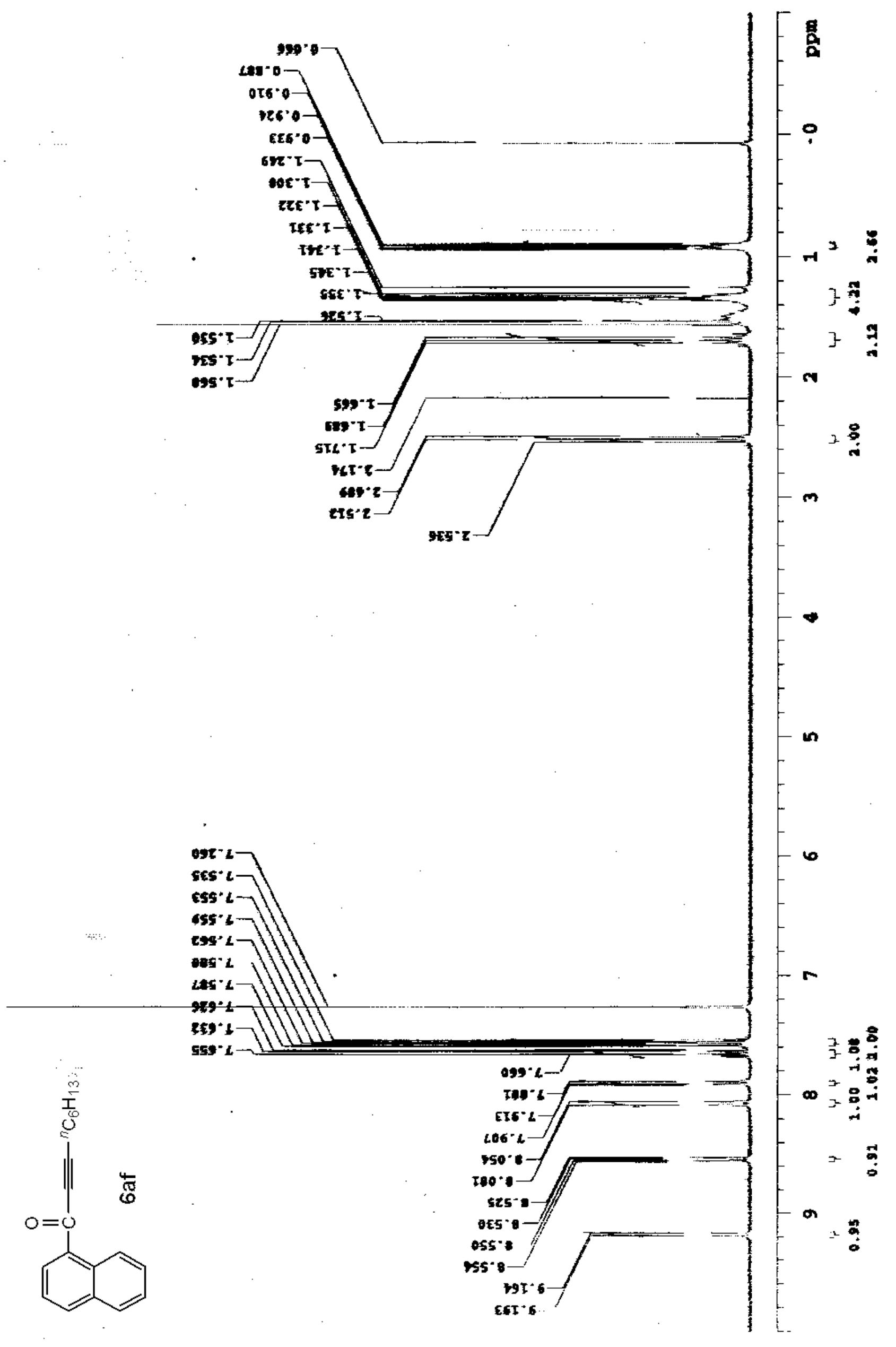




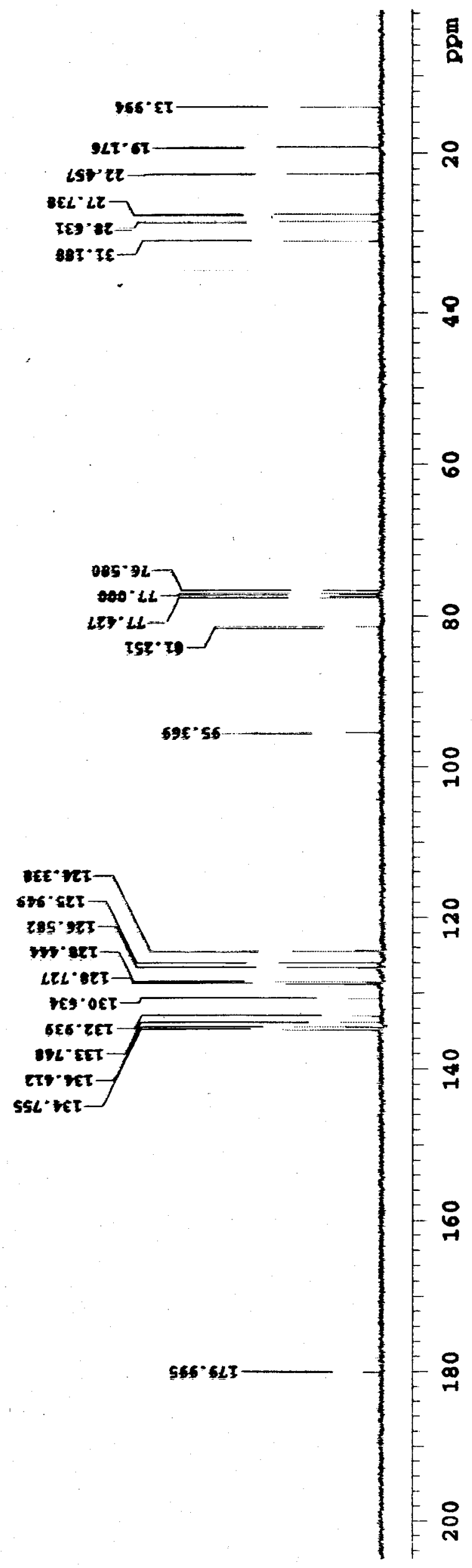




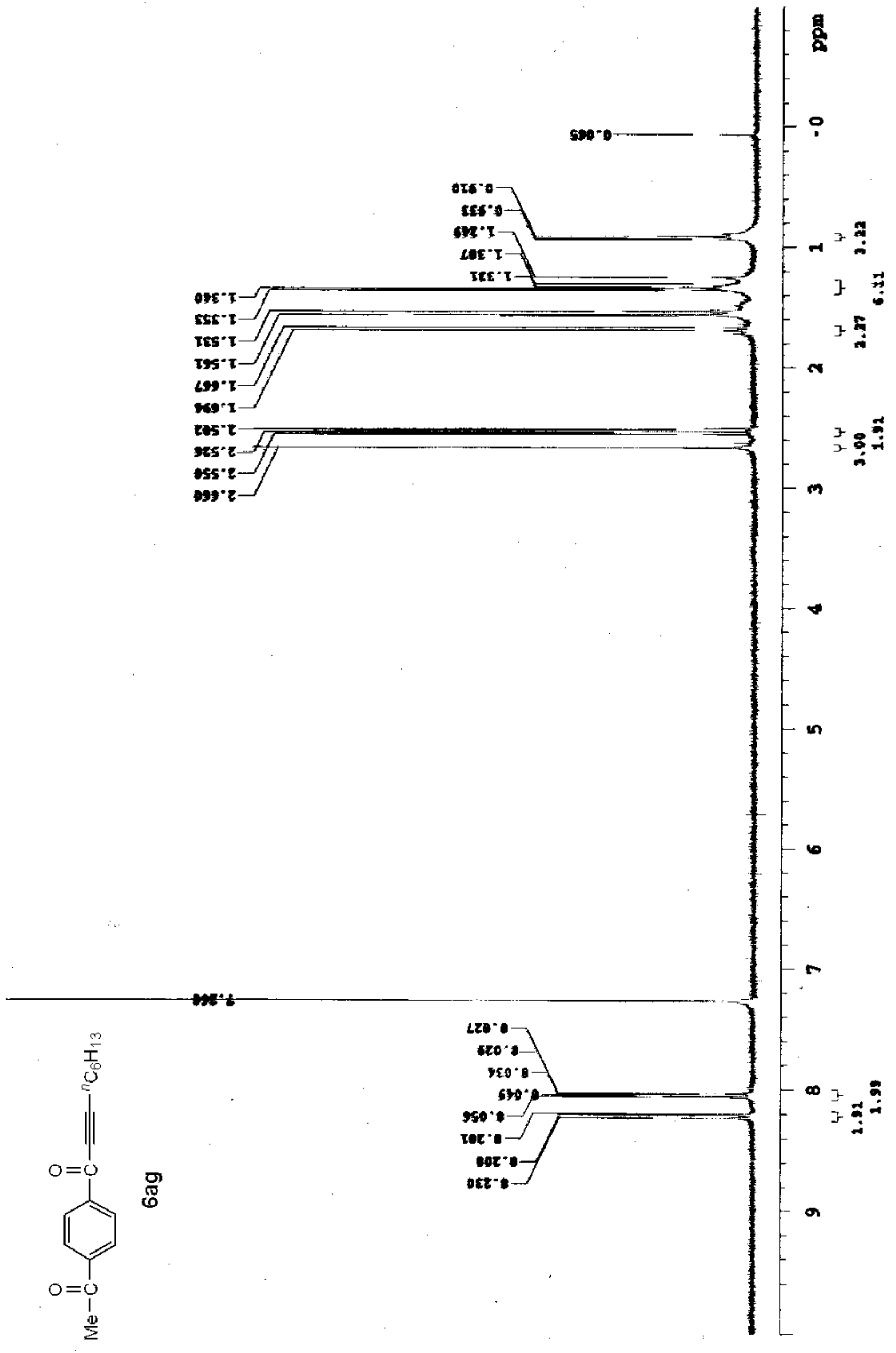




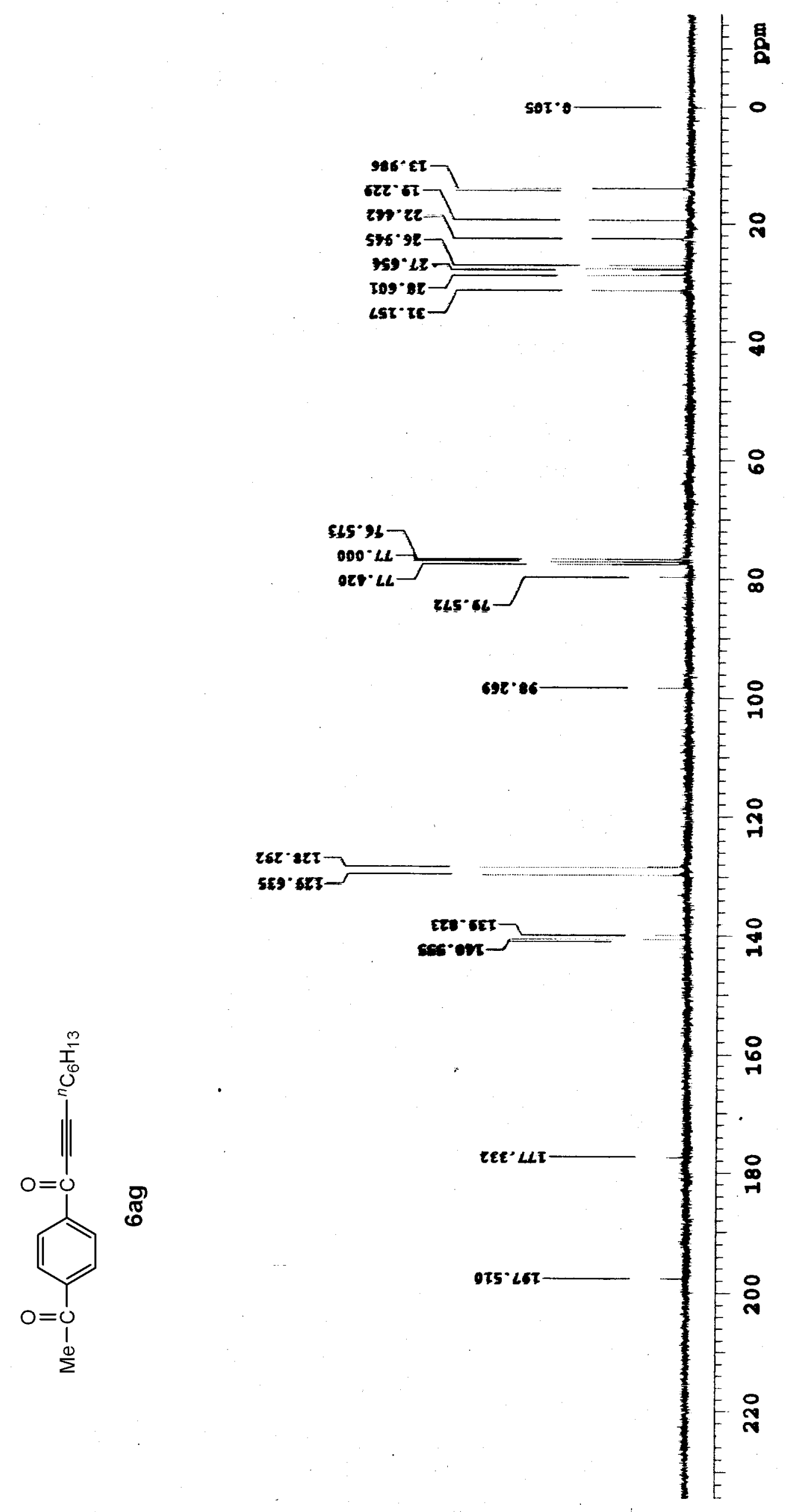




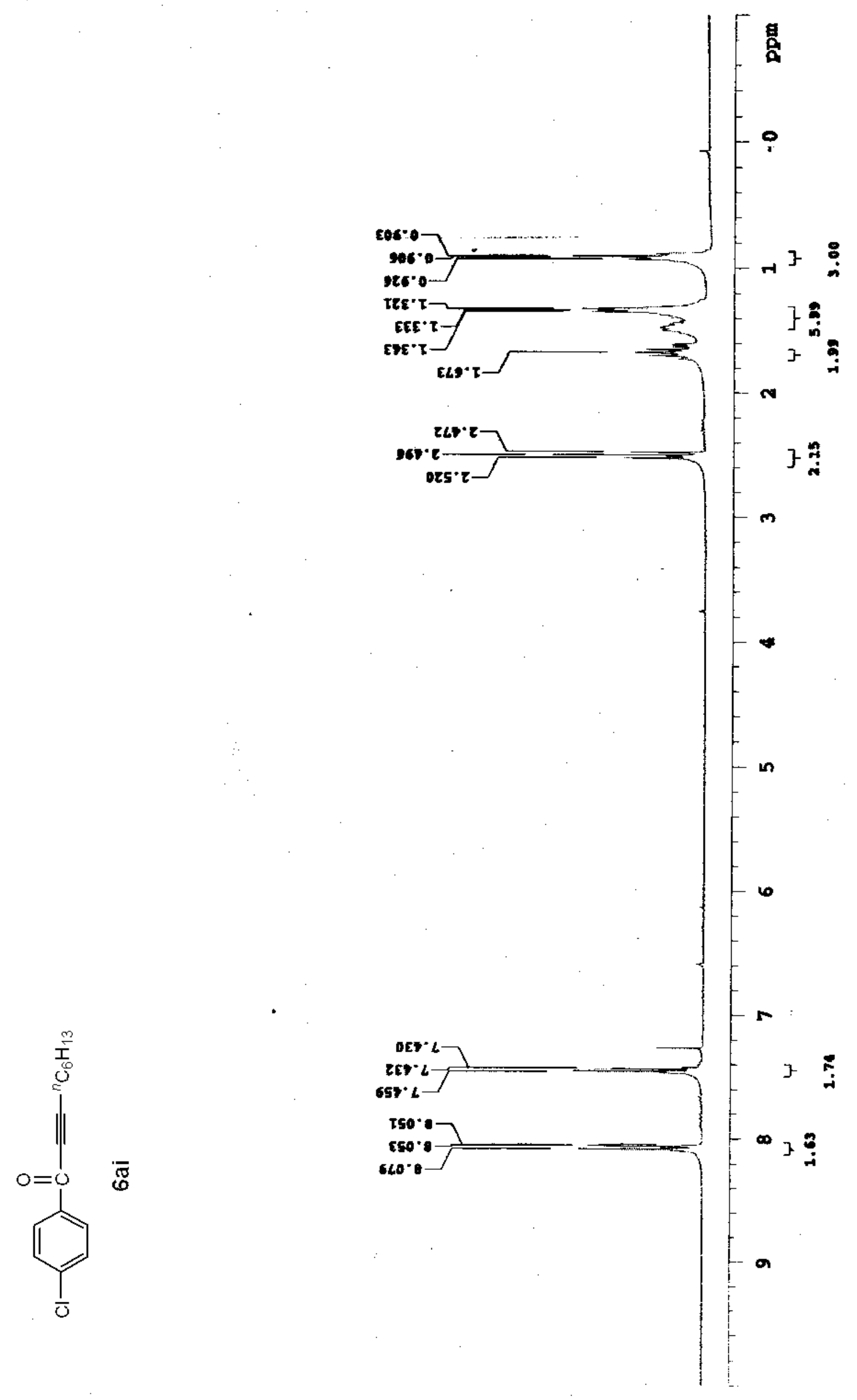




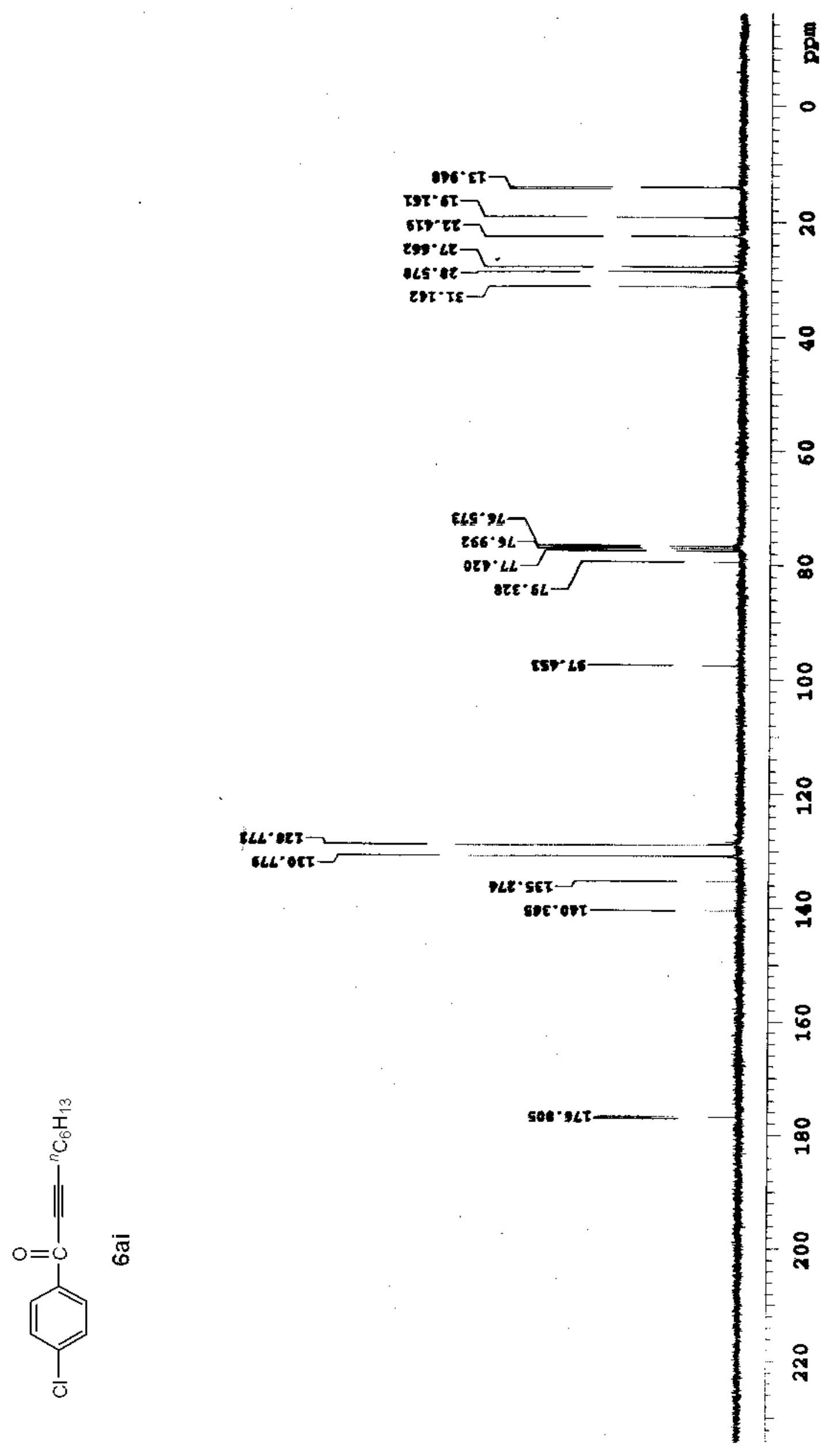




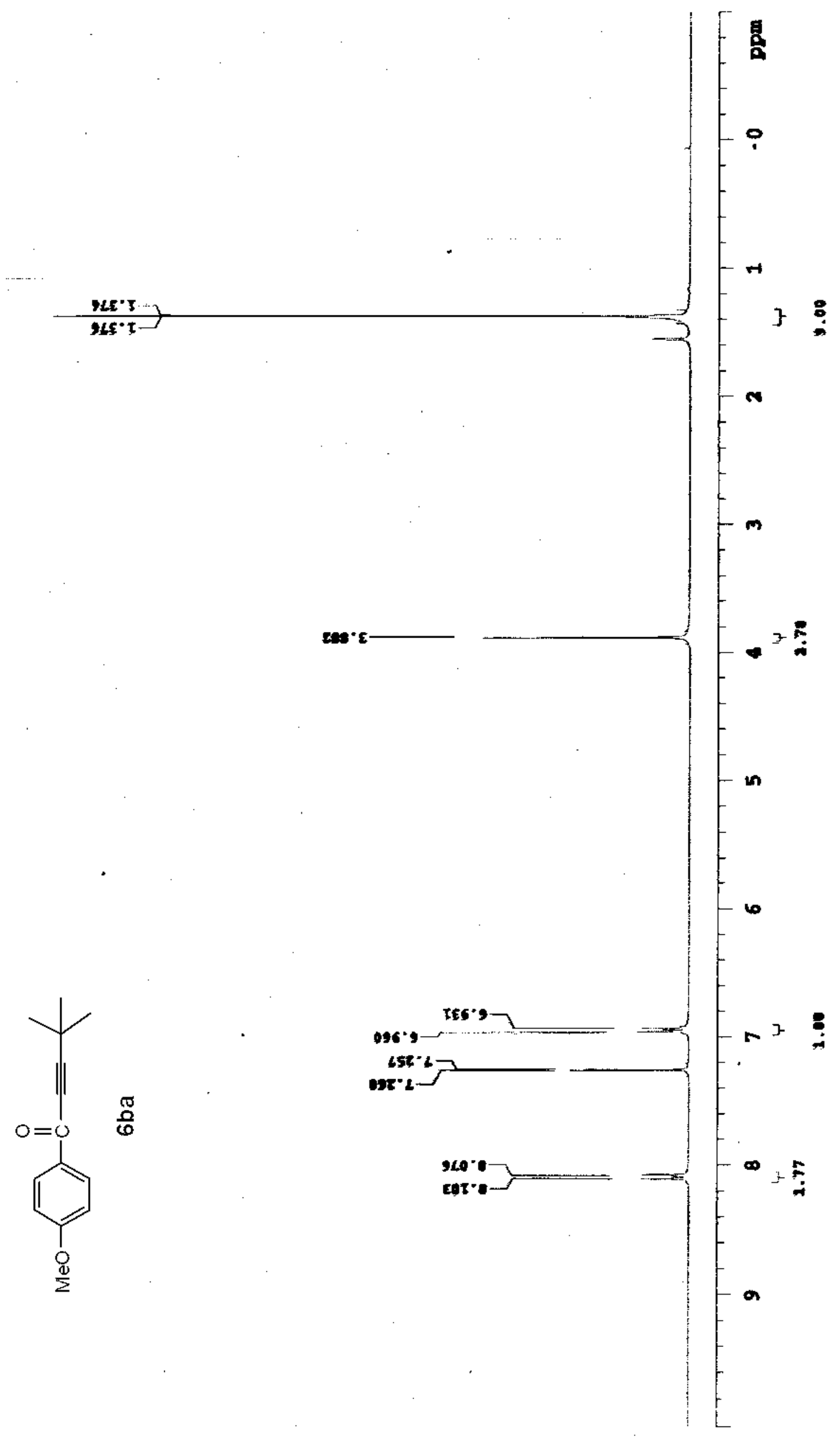




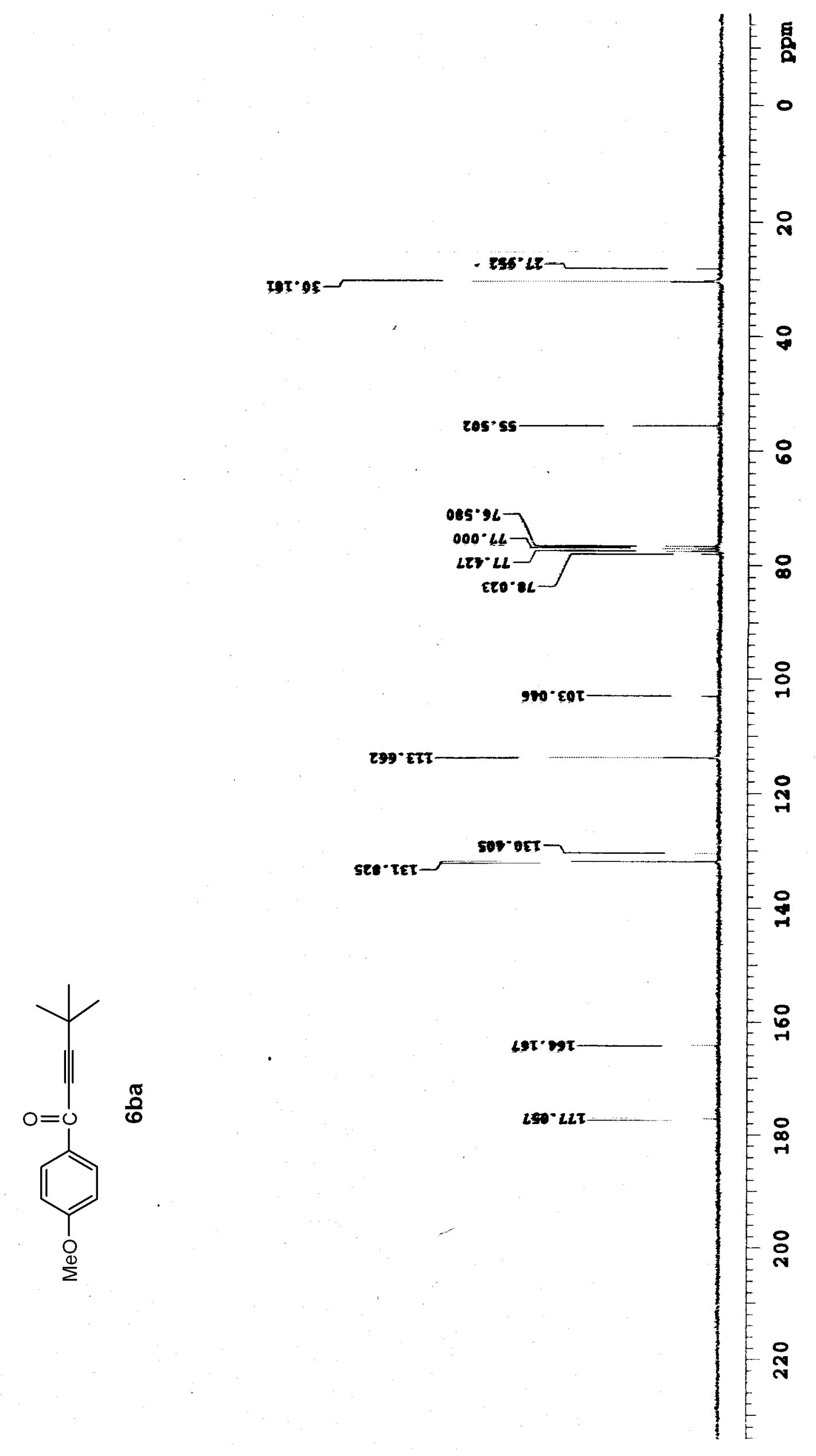




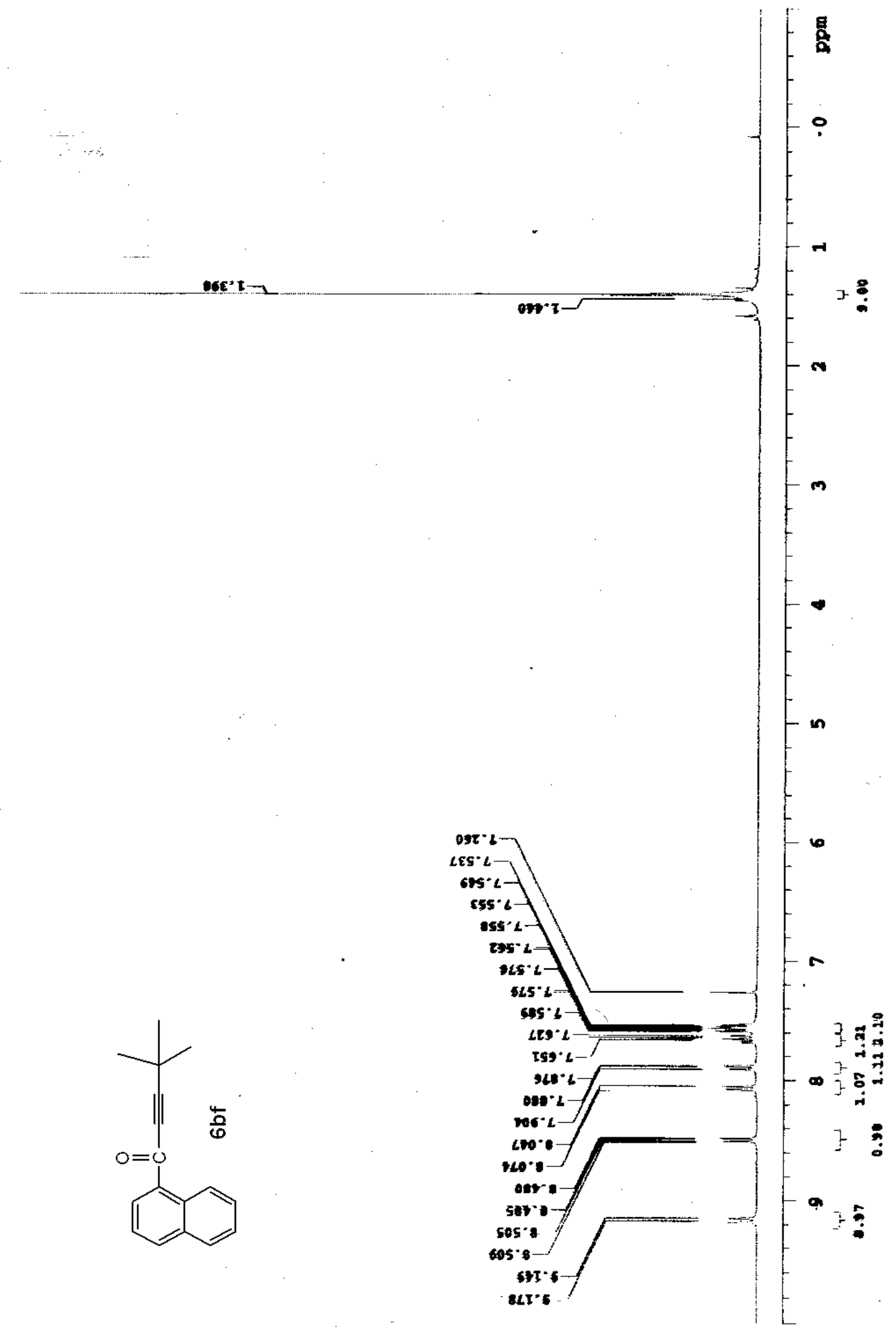




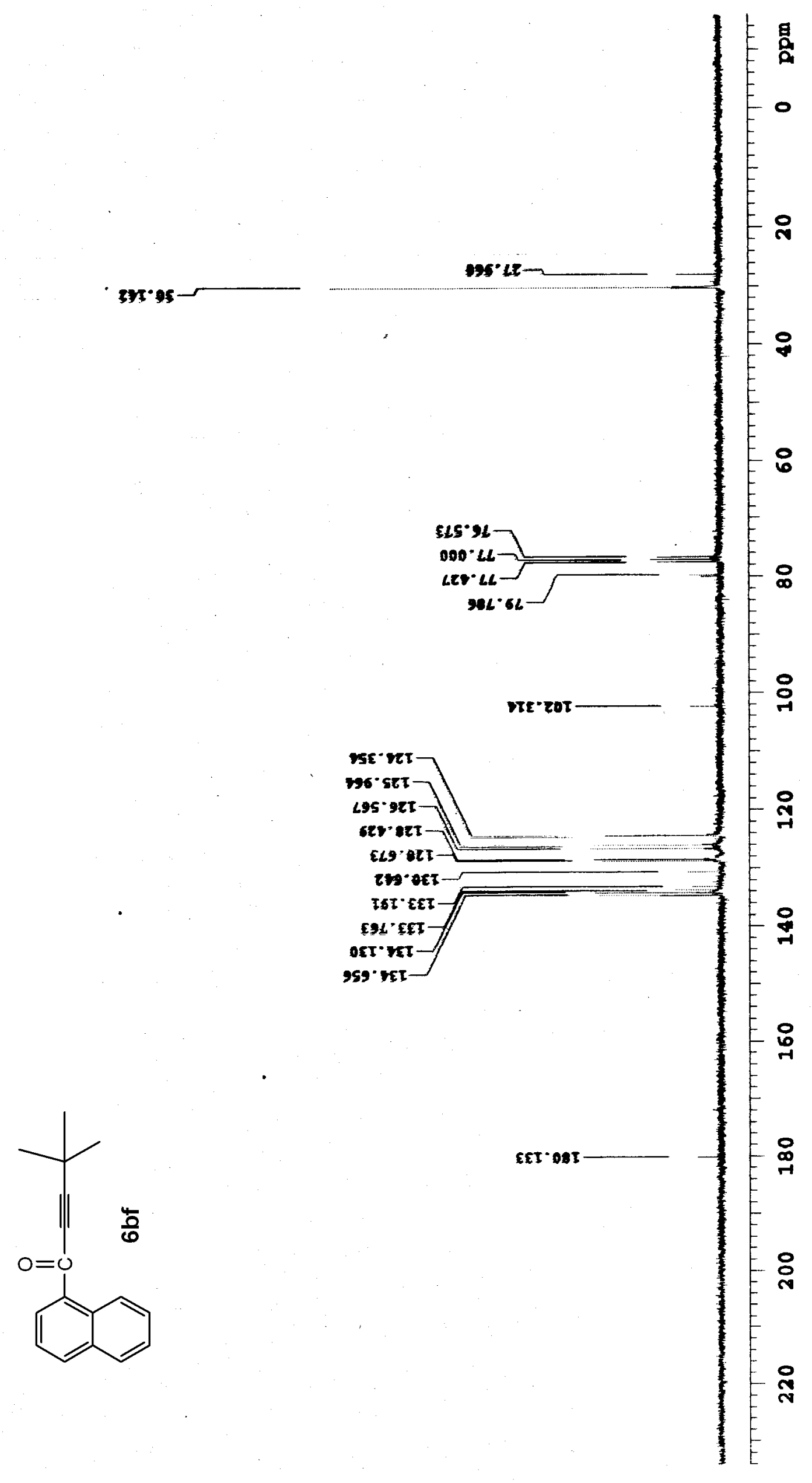




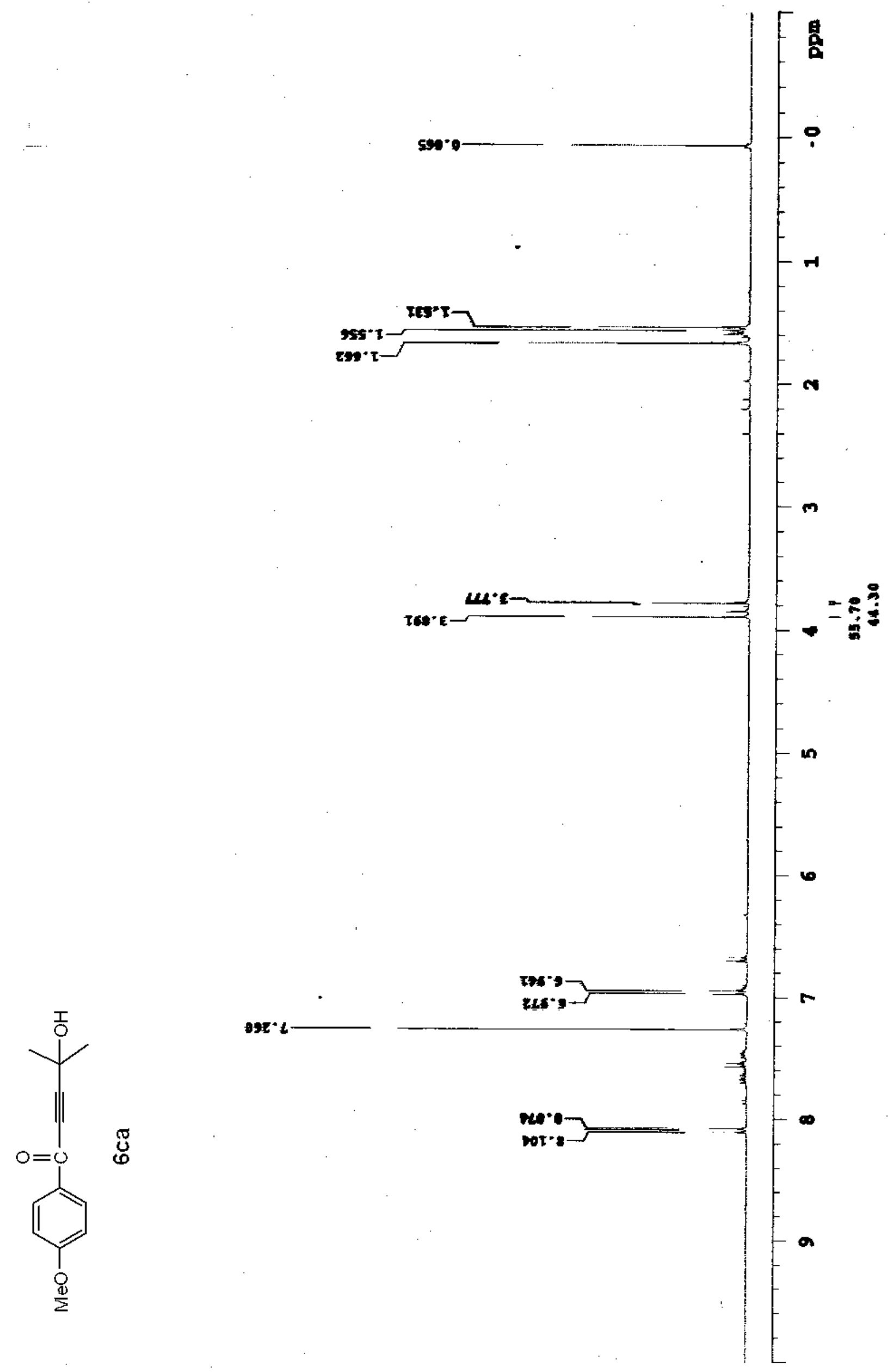




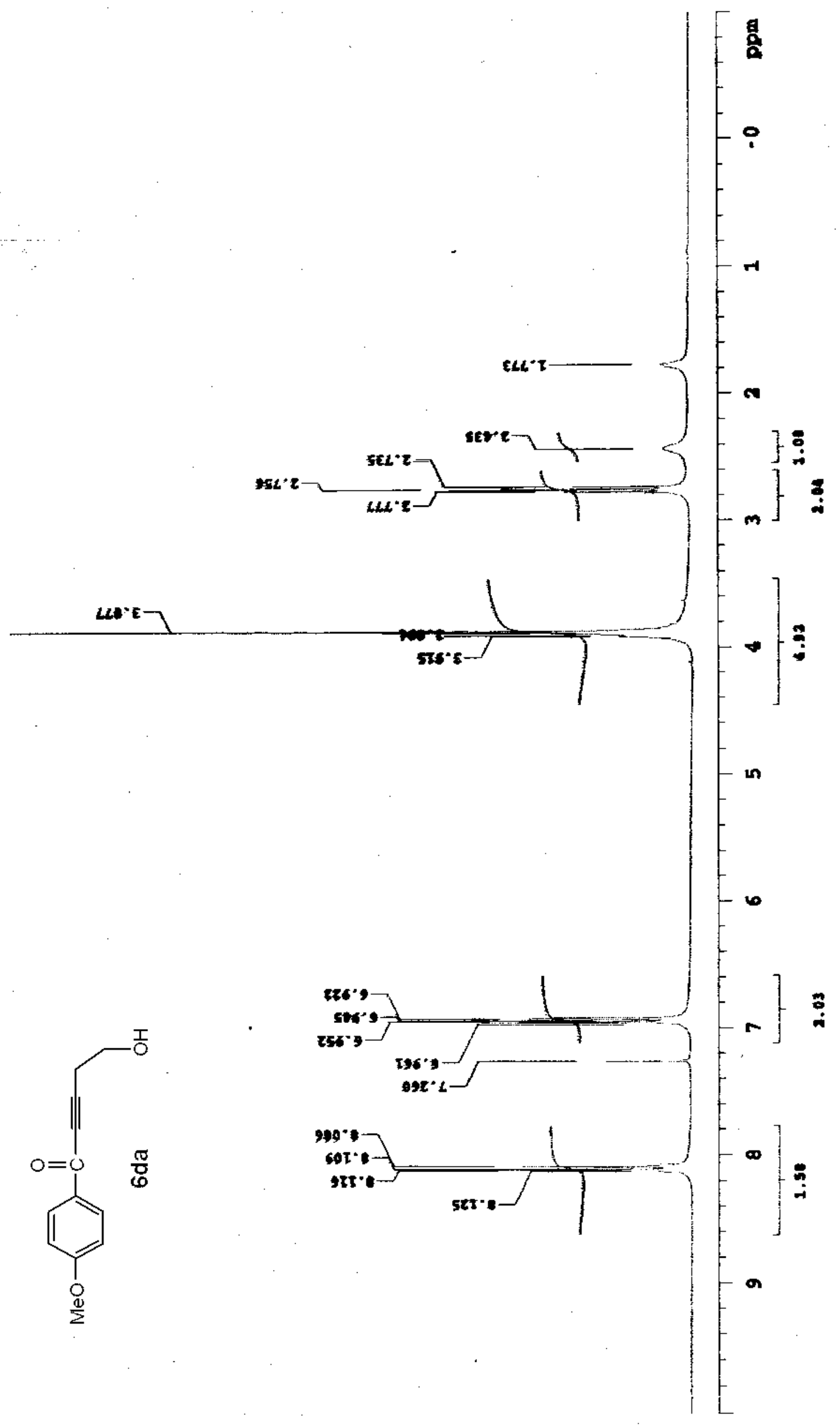




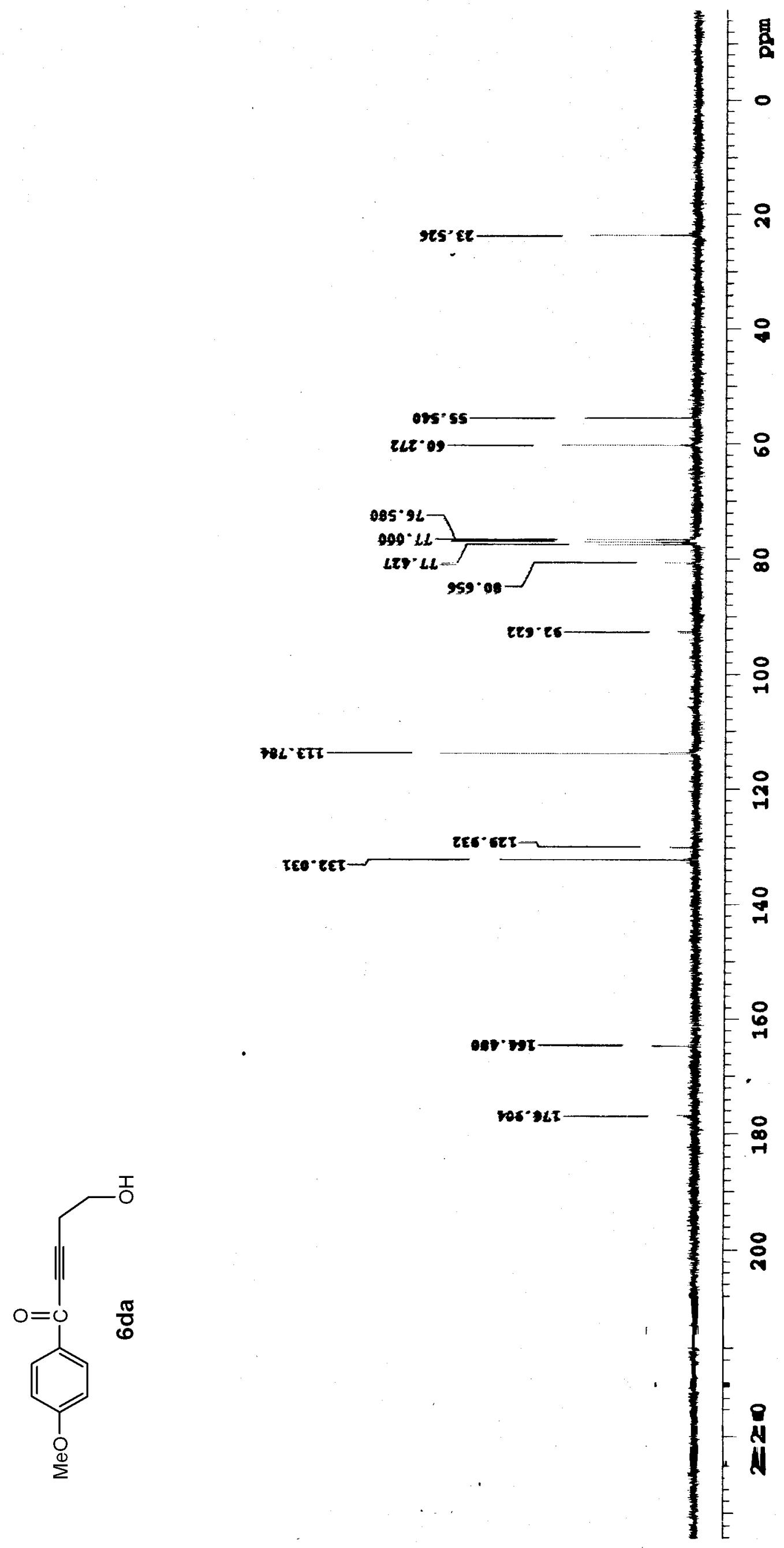




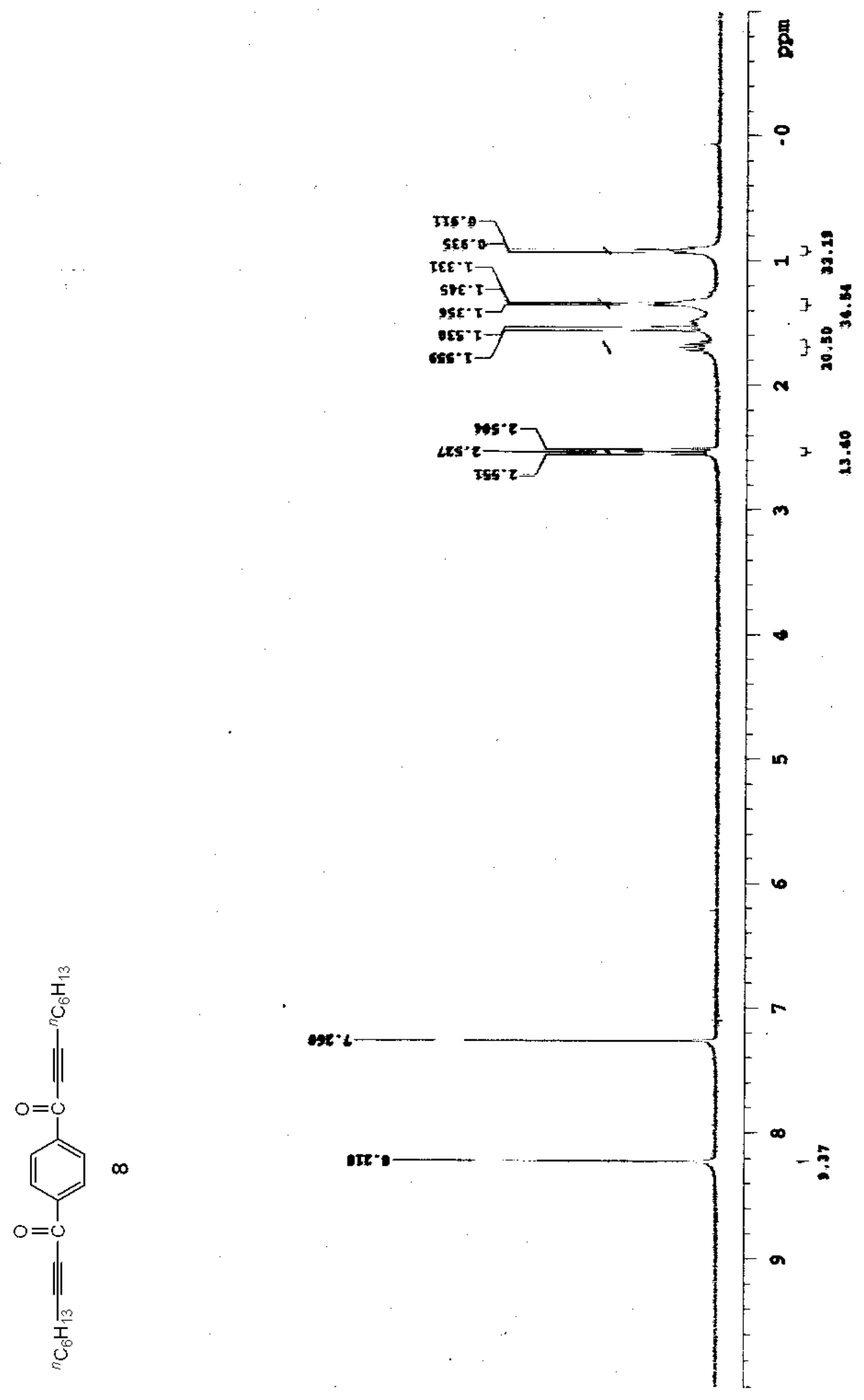




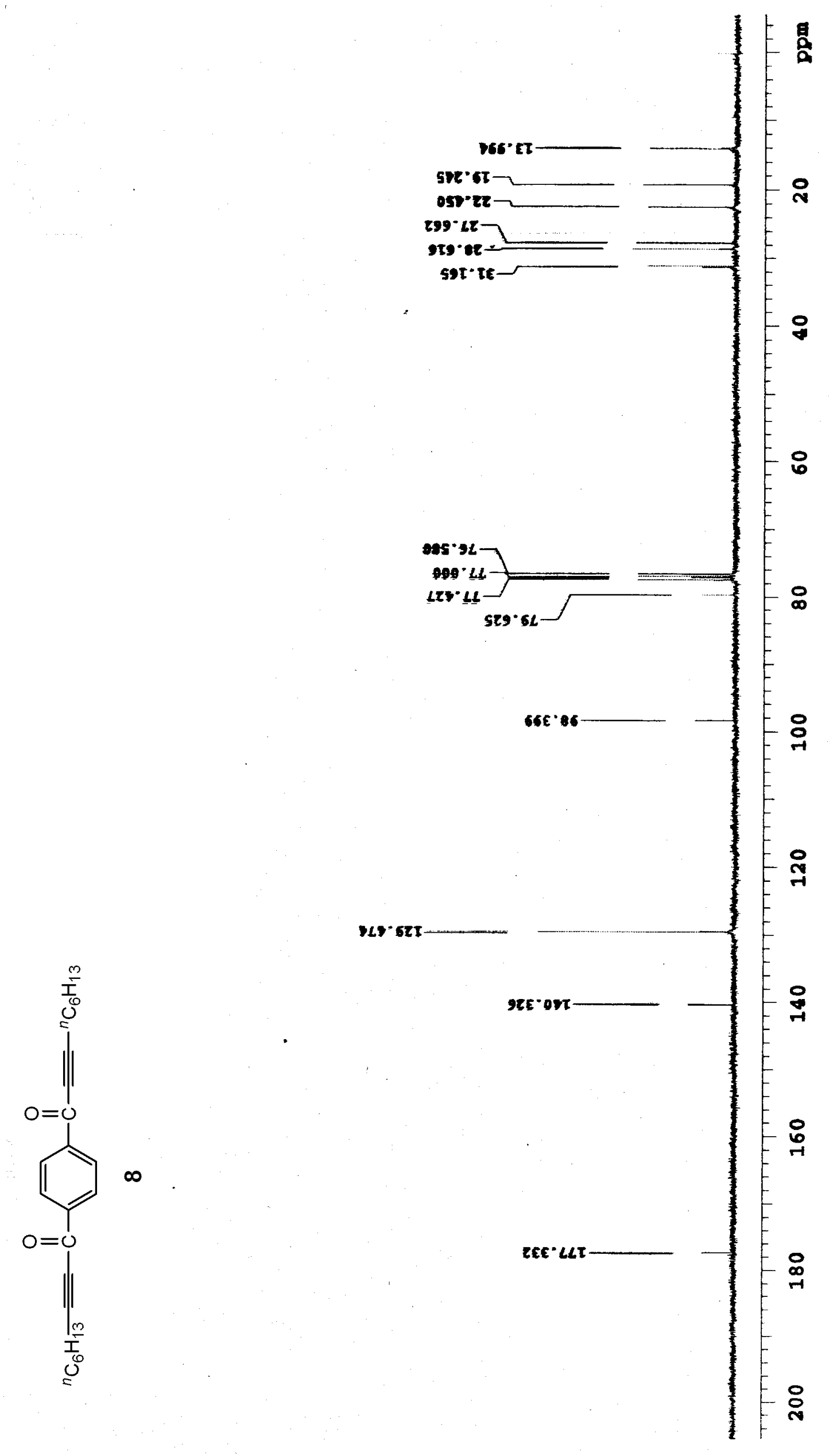




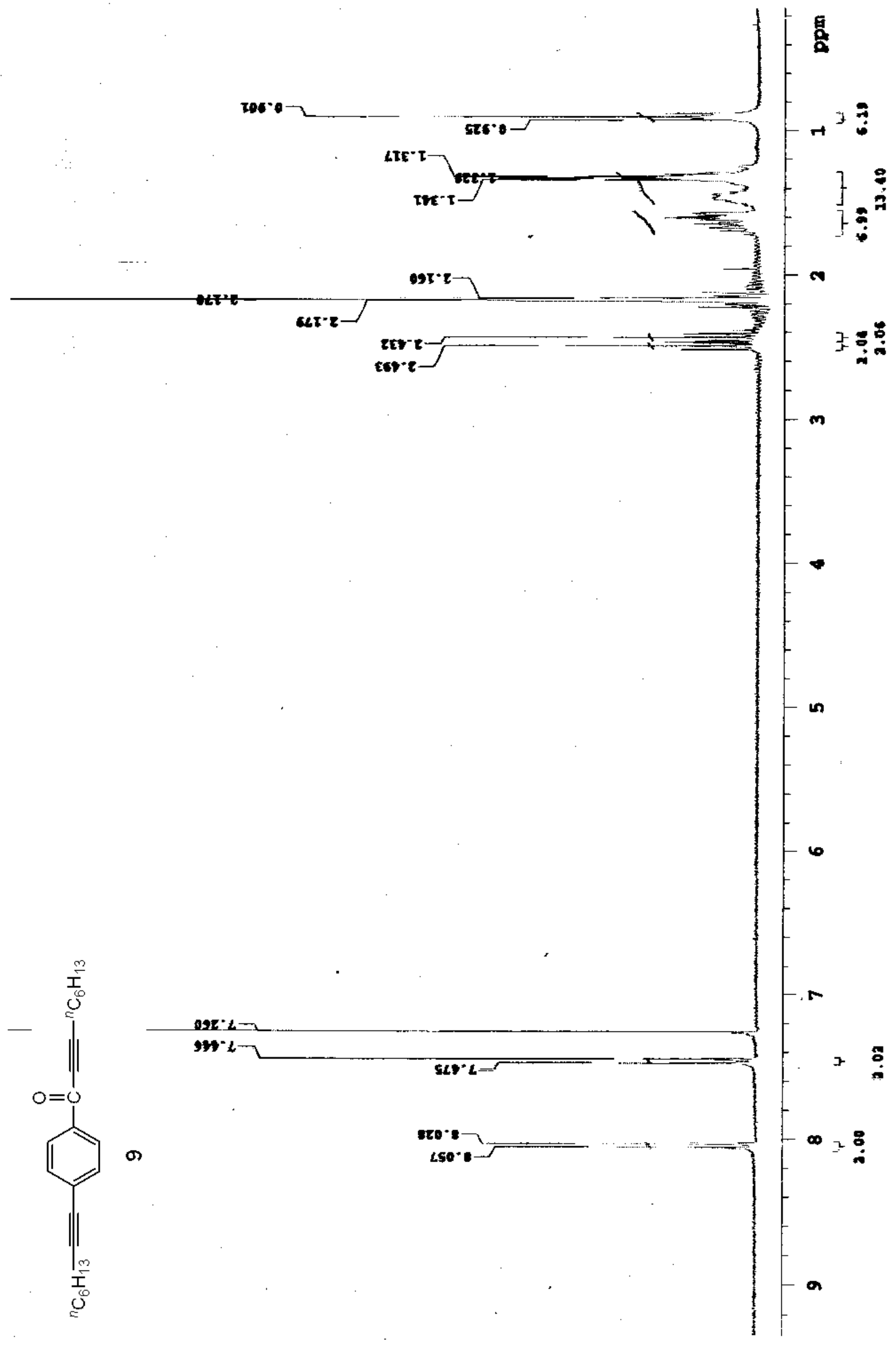




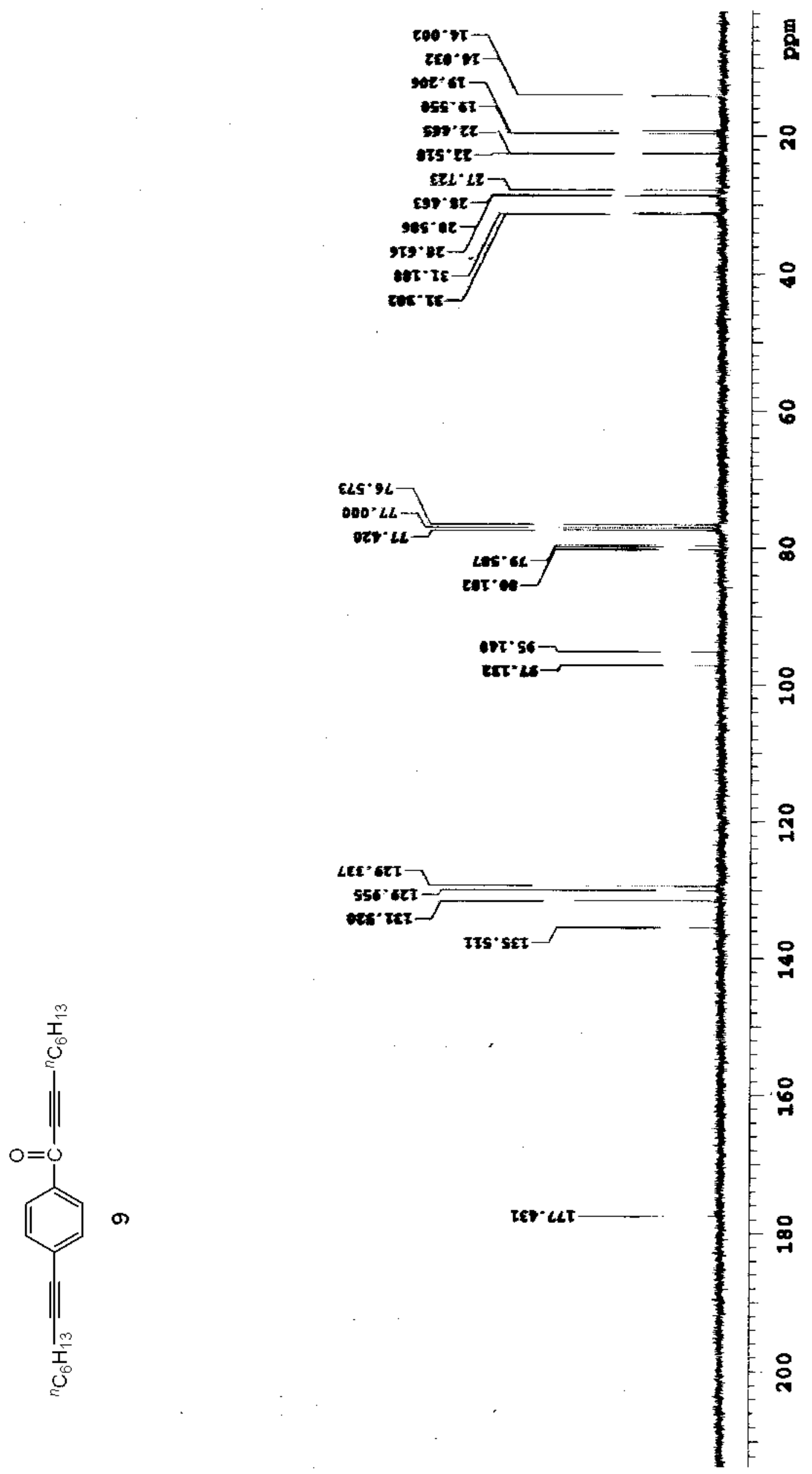

